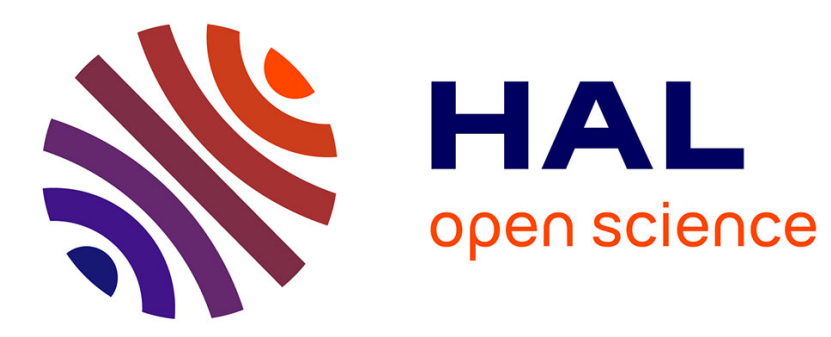

\title{
Novel approaches to develop biomarkers predicting treatment responses to TNF-blockers
}

Ikram Mezghiche, Hanane Yahia-Cherbal, Lars Rogge, Elisabetta Bianchi

\section{To cite this version:}

Ikram Mezghiche, Hanane Yahia-Cherbal, Lars Rogge, Elisabetta Bianchi. Novel approaches to develop biomarkers predicting treatment responses to TNF-blockers. Expert Review of Clinical Immunology, 2021, 17 (4), pp.331-354. 10.1080/1744666X.2021.1894926 . pasteur-03258281

\section{HAL Id: pasteur-03258281}

\section{https://hal-pasteur.archives-ouvertes.fr/pasteur-03258281}

Submitted on 11 Jun 2021

HAL is a multi-disciplinary open access archive for the deposit and dissemination of scientific research documents, whether they are published or not. The documents may come from teaching and research institutions in France or abroad, or from public or private research centers.
L'archive ouverte pluridisciplinaire HAL, est destinée au dépôt et à la diffusion de documents scientifiques de niveau recherche, publiés ou non, émanant des établissements d'enseignement et de recherche français ou étrangers, des laboratoires publics ou privés.

\section{(ㅇ)(1) $\$$}

Distributed under a Creative Commons Attribution - NonCommercial - NoDerivatives $\mid 4.0$ 


\section{Review}

\section{Novel approaches to develop biomarkers predicting treatment responses to TNF- blockers.}

Ikram Mezghiche ${ }^{\mathrm{a}, \mathrm{b}}$, Hanane Yahia-Cherbal ${ }^{\mathrm{a}, \mathrm{c}}$, Lars Rogge ${ }^{\mathrm{a}, \mathrm{d},{ }^{*}}$ and Elisabetta Bianchia ${ }^{\mathrm{a}, \mathrm{d}}{ }^{*}$

a Immunoregulation Unit, Institut Pasteur, Department of Immunology, Paris, France.

${ }^{b}$ Université de Paris, Sorbonne Paris Cité, Paris, France.

${ }^{\mathrm{C}}$ Fondation AP-HP, Paris, France.

d Unité Mixte AP-HP/Institut Pasteur, Institut Pasteur, Paris, France.

* Corresponding authors: Elisabetta Bianchi, MD

Institut Pasteur, Immunoregulation Unit, Department of Immunology

25, rue du Dr. Roux, 75724 Paris, France

Phone: +33-1-40613827, mail: elisabetta.bianchi@pasteur.fr

Lars Rogge, PhD

Institut Pasteur, Immunoregulation Unit, Department of Immunology

25, rue du Dr. Roux, 75724 Paris, France

Phone: +33-1-40613822, mail: lars.rogge@pasteur.fr

ORCID ID: 0000-0003-1262-9204 


\section{ABSTRACT}

\section{Introduction}

Chronic inflammatory diseases (CIDs) cause significant morbidity and are a considerable burden for the patients in terms of pain, impaired function and diminished quality of life. Important progress in CID treatment has been obtained with biological therapies, such as Tumor-Necrosis-Factor blockers. However, more than a third of the patients fails to respond to these inhibitors, and are exposed to the side effects of treatment, without the benefits. Therefore, there is a strong interest to develop tools to predict response of patients to biologics.

\section{Areas covered}

We searched PubMed for recent studies on biomarkers for disease assessment and prediction of therapeutic responses, focusing on the effect of TNF blockers on immune responses in Spondyloarthritis (SpA), and other CID, in particular rheumatoid arthritis and inflammatory bowel disease. Conclusions will be drawn about the possible development of predictive biomarkers for response to treatment.

\section{Expert opinion}

No validated biomarker is currently available to predict treatment response in CID. New insight could be generated through the development of new bioinformatic modelling approaches to combine multidimensional biomarkers that explain the different genetic, immunological and environmental determinants of therapeutic responses.

KEYWORDS: spondyloarthritis, ankylosing spondylitis, chronic inflammatory disease, rheumatoid arthritis, inflammatory bowel disease, anti-TNF therapy, biomarkers, treatment response, prediction, transcriptome, immune responses

\section{HIGHLIGHTS}

- Anti-TNF therapy has strong effects on several immune response pathways, modulating gene expression, cell population frequencies and serum protein levels.

- Several biomarkers for disease progression in patients undergoing anti-TNF treatment have been identified, however no biomarker has been validated for clinical use to predict response to treatment at baseline.

- Genetic biomarkers based on single nucleotide polymorphisms have demonstrated limited power to predict response to treatment.

- The combination of several biomarkers may improve the prediction power of statistical models of response to anti-TNF therapy. In particular, the inclusion of different types of biomarkers (genetic, 
transcriptional, protein, cellular) may be necessary to capture the biological complexity of response to treatment.

- New bioinformatic tools, including machine learning approaches, are necessary to handle the complexity of the large data sets being currently explored.

\section{Introduction}

Chronic inflammatory diseases (CID), such as inflammatory bowel disease (IBD), rheumatoid arthritis (RA), spondyloarthritis (SpA), psoriatic arthritis (PsA), and psoriasis (Pso) are a leading cause of morbidity. These conditions are associated with chronic pain and important functional impairment that have a large impact on productivity and quality of life $[1,2]$.

In this review we will focus mainly on Spondyloarthritis $(\mathrm{SpA})$, but also draw parallels with the larger literature on RA and IBD. SpA is one of the most common chronic inflammatory rheumatic diseases, with a prevalence ranging from $0.5 \%$ to $1.9 \%$ [3]. In addition to the disabling rheumatic manifestations, some SpA patients develop severe extra-articular manifestations such as inflammatory bowel disease (IBD), uveitis and psoriasis (Pso) [4]. SpA mainly affects young adults and the functional consequences of inadequately controlled disease alter both their quality of life and their professional capacity with direct impact on healthcare costs [5]. Improved control of the disease for affected individuals is thus an important public health issue. The introduction of biological therapies, such as strategies targeting the proinflammatory cytokine TNF, has revolutionized the treatment of CID. However, 30 to $40 \%$ of the patients fail to respond or acquire resistance to TNF-blockers, and it is currently not possible to predict response of patients to anti-TNF therapy [6-8]. Recently, anti-IL-17A drugs have been approved as a biologic alternative for axial $\mathrm{SpA}(\mathrm{axSpA})$ patients with active disease that do not respond to TNF inhibitors (TNFi), but this treatment also fails in about a third of the patients [9].

As a consequence, in current clinical practice treatment failures are observed for a substantial number of patients, who will not be appropriately treated for several months, while being exposed to the potential side effects of the drugs. There is therefore an urgent medical need to develop tools to guide treatment decisions for patients affected by SpA and other CID. The ability to predict the response to biologics, and to optimize the treatment will be the challenge for the next decade in CID.

Thus far, clinicians do not know, prior to treatment initiation, if a patient will respond or not to the treatment. Despite many efforts to identify predictive biomarkers of anti-TNF treatment response, there is still an unmet need for approaches that permit a pretreatment stratification of patients resulting in better patient healthcare and significant socio-economic benefits [9]. 
In this review we will explore recent work on the mechanistic action of TNF blockade in SpA, and comment on the latest approaches to identify predictive biomarkers. Comparison will be drawn with other CID (IBD and RA) for which a rich literature is available.

We performed an extensive literature search using PubMed. Our search mainly focused on papers published between 2015 and December 2020, but a few previous papers relevant to our subject were also included. We used keywords such as "Spondylarthritis", "Rheumatoid Arthritis", "Inflammatory Bowel Disease", "Anti-TNF $\alpha$ " (and single anti-TNF drugs), "Biomarkers", "Outcome", "Response" and "Prediction". Since the literature on predictive biomarkers is still limited, we also included papers investigating biomarkers associated with disease activity. Only English written papers were considered for our review.

\section{Effects of anti-TNF therapy on the immune system in patients - a brief summary}

The introduction of anti-TNF therapy has been a major breakthrough for the treatment of several chronic inflammatory diseases, in particular rheumatoid arthritis [10] (reviewed in [11]), inflammatory bowel disease [12,13], ankylosing spondylitis [14](reviewed in [15]), and psoriatic arthritis and psoriasis $[16,17]$. Early mechanistic studies revealed a strong reduction of inflammatory cytokines and acute phase proteins, such as IL-1, IL-6 and CRP within hours after injection of a TNF inhibitor (TNFi) [18]. Furthermore, levels of inflammatory chemokines and VEGF were also reduced, causing reduced granulocyte recruitment and angiogenesis in arthritic joints [19,20] (reviewed in [21]).

RNA sequencing technologies have become relatively affordable and permit the assessment, on a genome-wide level, of biomarkers within immune cells that might not be detectable in serum. mRNA profiling in SpA patients before and after treatment onset, may help identify molecular pathways associated with response to therapy. Using RNA sequencing, a gene expression analysis was performed on nineteen AS patients, to profile the transcriptomes of peripheral blood cells (PBMCs), and identified 656 genes differentially expressed before and after anti-TNF treatment. Analysis of signaling pathways using KEGG (Kyoto Encyclopedia of Genes and Genomes) revealed an enrichment of several immune and inflammation regulatory pathways, as well as infection metabolism-associated pathways in genes affected by anti-TNF therapy [22]. Among the differentially expressed genes were also genes associated to AS in GWAS studies (IL6R, NOTCH1, IL10, CXCR2 and TNFRSF1A), highlighting pathogenic pathways that may be affected by TNF signaling. The altered expression of components of the NOTCH signaling pathway found in AS may be involved in osteoblast differentiation and ossification processes [23]. TNFRSF1A encodes an important receptor for TNF, and SNPs in the locus of the anti-inflammatory cytokine IL-10 have shown modest correlation with disease severity [24]. Interestingly, the study showed a distinct gene expression profile between male and female patients. Besides genes linked to sex chromosome, the only differentially expressed gene was IL17RC, which encodes an IL-17 receptor that binds IL-17A and IL-17F. This 
proinflammatory pathway may play an important role in SpA [25]. An interesting hypothesis is that higher expression of IL17RC may be involved in the differences in disease severity and treatment response between the genders [22].

It is important to note that most transcriptomic studies are performed in PBMCs, and the mixture of different cell populations may hide biologically important features specific to a cell type. Peripheral blood cells may also not be representative of local inflammatory processes [26], however, biomarkers that can be identified in peripheral blood are worth investigating, since they are more easily applicable in large scale studies and in the clinics.

Menegatti et al. have recently investigated the global impact of TNFi on immune responses to microbial or pathway-specific stimuli in axSpA patients. The motivation for this study was that the effects of TNF-blockers had been studied mostly on resting immune cells but not in the setting of an ongoing immune response. The goals were to enhance the understanding of the molecular mechanisms of action of TNF-blockers in SpA patients and to identify immunological correlates of response to TNFi. To minimize sources of pre-analytical variability the authors used standardized whole-blood stimulation assays ("TruCulture" assays) [27] and a highly sensitive and robust pipeline to assess immune functions in patients [28]. Proteins in supernatants of stimulated whole-blood cultures were measured in a CLIA-certified laboratory and gene expression was measured using nCounter assays for immune genes, a technology not requiring enzymatic reactions and PCR amplification, already used in the clinics for diagnostic purposes [29]. Up to 300 genes (depending on the stimulation) were affected by anti-TNF therapy, revealing that TNFi induce profound changes in patients' innate immune responses. Stimulation of whole blood amplified the observable differences between samples from patients before and after TNF-blocker treatment, indicating that TNFi act primarily when the immune system is challenged but less in its resting state. The effects of TNFi on activated immune cells were detectable after a single injection of a TNF-blocker and persisted for 3 months of follow-up of the patients.

To understand the molecular basis of anti-TNF therapy action on immune responses, pathway analysis was performed on the differentially expressed genes. Since nCounter technology does not allow a genome-wide gene expression analysis, the Quantitative Set Analysis for Gene Expression (QuSAGE) method was employed [30]. QuSAGE quantifies gene module activity as a shift in the mean differential expression of the individual genes included in the module and is compatible with a limited number of genes. Similar to other GSEA approaches, QuSAGE allows reducing the number of variables by collapsing sets of coordinately expressed genes into gene modules, facilitating functional interpretation of the results and improving robustness of biomarker signatures. Bancherau et al. have previously applied QuSAGE to functionally interpret gene lists associated with specific phenotypes in Lupus patients [31] and Latis et al. used this algorithm to compare and interpret $\mathrm{T}$ cell gene expression profiles in recipients after allogeneic hematopoietic stem cell transplantation and their sibling donors [32]. Menegatti et al. designed gene 
modules by grouping genes belonging to specific signaling pathways, associated with particular cellular phenotypes, or with specific cellular functions, according to Molecular Signatures Database (MSigDB) annotations [33] and based on current knowledge in the literature. Since genes can play roles in distinct signaling pathways, a single gene could be present in several modules. The minimum size of one module was set to 3 genes. Fig. 1 gives an example for the influenza stimulus. The TNF module groups TNF and several molecules associated with its receptor. TNF itself and many of the genes in this gene module were downregulated after one week of anti-TNF therapy. This is similar for the IL-1 module. In this study, IL1A was among the genes most strongly targeted by anti-TNF therapy. As for Toll-like-receptors (TLRs), some were downregulated, whereas the expression of others actually increased. Signaling through many of these receptors activates NF-kB regulatory kinases that phosphorylate the NF-kB inhibitor IkB and thereby activate NF-kB. NF-kB transcription factors, IkB and many NF-kB target genes were among the genes that were most profoundly downregulated by TNF blockers (Fig. 1 and 2). Since TNF itself, IL-1 and many other proinflammatory molecules are NF-kB target genes, one of the mechanisms by which TNFi control inflammation may be the disruption of an autoregulatory loop driven mainly by NF-kB [28].

Only few gene modules showed an increased pathway activity, such as the "cytotoxic molecules" module and the "NK cells" (Fig. 3A). An increased fraction of CD8+ T cells following anti-TNF treatment has also been recently described in RA patients [34]. The analysis also indicated that TNF-blockers may skew monocyte/macrophage polarization towards an M2 regulatory phenotype (Fig. 3B). M2-polarized macrophages are implicated in the resolution of inflammation, have immunoregulatory functions and orchestrate tissue repair and remodeling $[35,36]$. On the other hand, shifting the balance from a M1-like to a more M2-like profile may contribute to the increased risk of Mycobacterium tuberculosis reactivation in patients treated with TNFi [37-39], as M1 macrophages are important for granuloma formation and $M$. tuberculosis protection [40].

Anti-TNF treatment did not affect the Th1 or Th17 arms of the patients' immune response, nor IL-6 production in cells from axSpA patients, which contrasts previous results reported for RA [18]. These data seem consistent with the limited therapeutic efficacy of IL-6-blockade in SpA [41] and may suggest that IL6 is more relevant to RA, but less to SpA pathogenesis.

Collectively, the analysis by Menegatti et al. revealed that TNFi target several distinct signaling pathways that cooperate to control inflammation. Some of these pathways may be of particular relevance for specific diseases. PTGS2 (COX2) downregulation by TNFi (Fig. 3C) targets PGE 2 biosynthesis and is of particular relevance for enthesitis, a critical early pathogenic feature of SpA [42], while shifting the balance of macrophages from a pro-inflammatory (M1-like) phenotype to a pro-resolving (M2-like) phenotype is important for the resolution of synovitis, a key feature of RA. Expression of the PGE 2 receptor EP4 (encoded by PTGER4) was also downregulated by TNFi. Signaling through the EP4 receptor upregulates IL-23R expression and promotes human Th17 cell development [43]. 


\section{The search for biomarkers of response}

Anti-TNF therapy is not effective in $30-40 \%$ of patients affected by chronic inflammatory diseases, but the mechanisms underlying primary non-response to treatment are not known. Many studies have been performed to identify molecules that may serve as biomarkers to predict therapeutic responses to TNFblockers, in particular in RA, IBD, and SpA (reviewed in $[6,7,44,45]$ ), however, no validated biomarker has yet emerged. In the following, we will summarize recent studies aiming at the identification of biomarkers for response to treatment, in particular in SpA, RA and IBD.

\subsection{Transcriptomic biomarkers}

To determine if therapeutic responses to TNFi were correlated with immune responses from SpA patients, Menegatti et al. have analyzed gene expression in whole-blood cultures stimulated with LPS or SEB from $80 \mathrm{SpA}$ patients, before initiation of anti-TNF therapy [28]. The response to therapy was evaluated as changes in the "Ankylosing Spondylitis Disease Activity Score" (ASDAS) at 3 months after treatment initiation [46,47]. Gene expression analysis of these cultures revealed that 55 genes that were differentially expressed between responders and non-responders (Fig. 4) [28]. 15 of these genes were associated with leukocyte migration and invasion, such as the genes encoding urokinase (PLAU) and its receptor (PLAUR). The importance of leukocyte recirculation as a determinant of treatment responses to TNFi was supported by the finding that several chemokines and their receptors were differentially expressed between responders and primary non-responders. Genes encoding the receptors for the pro-inflammatory cytokines TNF, IL-6 and IL-1 were also expressed at higher levels in cultures from responders, as was expression of NLRP3, the gene encoding the intracellular sensor NOD-, LRR- and pyrin domain-containing protein 3, which plays an important role in the control of caspase-1-dependent processing of pro-IL-1 $\beta$ and IL-18 into active cytokines [48]. A polymorphism in the NLRP3 gene (rs4612666) was associated with primary response to anti-TNF therapy in 2 independent cohorts of Ulcerative Colitis (UC) and IBD patients. This polymorphism is associated with reduced NLRP3 expression [49]. Only 7 differentially expressed genes were expressed at higher levels in non-responders, including CXCL9 (encoding a chemoattractant for CXCR3-expressing Th1 and other cytotoxic cells) and IFNG, encoding the signature cytokine of Th1, CD8 ${ }^{+} T$ and NK cells. Bank et al. previously reported that a polymorphism in the IFNG gene (rs2430561) was significantly associated with effective anti-TNF primary response in Crohn's disease (CD) patients but not in ulcerative colitis (UC) patients. This variant is associated with decreased IFN- $\gamma$ level [50]. This result was confirmed in another study in UC patients, where, at baseline, responders had lower mucosal mRNA expression of IFN- $\gamma$ than non-responders [51]. In contrast, Rismo et al. reported that high mRNA expression of mucosal IFN- $\gamma$ and IL-17A in biopsies obtained before anti-TNF therapy was associated with 
successful therapy response in UC patients [52], suggesting that the role of IFN- $\gamma$ in this context needs further evaluation.

IL7R, the receptor for the homeostatic cytokine IL-7 was expressed at higher levels in stimulated immune cells from axSpA patients not-responding to TNFi [28](Fig. 4). A 20 genes signature of IL-7/IL-7R signaling has recently been analyzed in colon mucosal biopsies from previously published cohorts of IBD patients [53]. This study revealed that the IL-7R signaling signature is reproducibly altered in inflamed mucosa, with a significant accumulation of $I L 7 R$ transcripts that may contribute to the maintenance of chronic inflammation. More interestingly, it also demonstrated that a strong colonic IL-7R signaling gene signature (and in particular IL7R), before initiation of therapy, is significantly and reproducibly associated with the absence of response to anti-TNF therapy both in UC and CD patients, identifying refractory IBD patients. The author showed that this is not the case for mucosal ileal biopsies, suggesting a specific association of the IL-7/IL-7R pathway with colonic but not ileal IBD inflammation. A combination of 10 genes (IL7R, IL2RG, JAK1, PIK3CA, LCK, PTK2B, EP300, NMI, CRLF2, and TSLP) from the IL-7R signaling signature was sufficient to discriminate anti-TNF non-responders from responders, constituting a potential new predictive biomarker to identify refractory patients [53]. A more recent IBD study developed a test to measure IL-7, together with 13 additional proteins in blood (ANG1, ANG2, CRP, SAA1, IL-7, EMMPRIN, MMP1, MMP2, MMP3, MMP9, TGFA, CEACAM1, and VCAM1). This signature was termed the "endoscopic healing index" (EHI). The EHI was used to score endoscopic disease activity in patients with $\mathrm{CD}$. The EHI scores range from 0 to 100 units, with higher scores indicating more severe CD activity, based on endoscopy findings. Outcome prediction based on EHI was comparable to measurement of FC (fecal calprotectin) and higher than measurement of serum CRP [54].

Transcriptomic biomarkers have also been investigated in RA, with some limited results [55]. In this respect, the combined analysis of gene expression and genetic data in large patient cohorts may be a more promising approach. Aterido et al. defined gene co-expression modules in RA synovial tissue and analyzed their association with response to anti-TNF treatment at the clinical and genetic level, using setbased genetic association analysis. The analysis resulted in the identification of an 18-gene module expressed in synovial tissue, and significantly associated at the genetic level with response to adalimumab [56]. Pathway analysis showed that the module is enriched for genes involved in nucleotide metabolism, which plays an essential role in cell proliferation and in the synthesis of signaling molecules such as adenosine. Notably, adenosine signaling in macrophages has been shown to induce a switch from a proinflammatory $\mathrm{M} 1$ phenotype to an $\mathrm{M} 2$ regulatory phenotype $[57,58]$.

Cherlin et al. applied the PrediXcan algorithm on genetic data from the MATURA consortium [59] to identify genes that are associated with changes in the erythrocyte sedimentation rate (ESR) in patients treated with TNF blockers. The prediction model identified the IL18RAP gene as a predictor of changes in ESR after treatment [60] . In a replication cohort, the expression of IL18RAP in whole blood at baseline 
correlated with changes in ESR at 6-month follow-up, and a correlation was observed between IL18RAP expression in blood and in synovial tissue [60]. IL18RAP is important for IL-18 signaling, a pathway implicated in inflammation, and a potential treatment target in RA [61]. The association of IL18RAP with treatment outcome is not specific to one drug type, making it an interesting biomarker to explore for treatment responses in RA.

In IBD patients, analyses of gene expression in inflamed tissue and immune cells also proved useful in the understanding of the immunopathogenesis of IBD and prediction of therapy outcome. In a study by Arijs et al. baseline gene expression levels in mucosal biopsies could distinguish responders from nonresponders to infliximab. The microarray analysis identified a set of 5 genes (TNFAIP6, S100A8, IL11, GOS2, and S100A9) that could be used to predict the response to anti-TNF treatment with $100 \%$ accuracy [62]. A subsequent genetic study demonstrated that haplotype differences in the loci of these 5 genes differentiated responders from non-responders to infliximab [63]. The same group identified also a panel of 5 genes that can be used to predict response to anti-TNF treatment in UC patient (OPG, STC1, PTGS2, IL13RA2, IL11) [64].

\subsection{Genetic biomarkers}

Explorative studies of genetic biomarkers to predict anti-TNF therapeutic outcome use genotyping technologies in order to associate genotype with good or bad response to treatment. Genotyping has been performed either on a genome-wide scale (GWAS), or on a narrower scale, by targeting a specific set of single nucleotide polymorphisms (SNPs), selected for their previous association with disease susceptibility or with proposed correlation with anti-TNF response. Results from GWAS performed on rheumatic diseases suggest that disease susceptibility and treatment response are influenced by the action of many genetic variants with modest effects, rather than a few variants with large effects. Thus, polygenic risk scores, based on the combination of several potentially predictive gene variants are expected to be more efficient in predicting treatment response than individual risk variants $[65,66]$.

Overall, genetic biomarkers are particularly interesting as early predictive biomarkers: they are invariable, they precede disease onset and use affordable technologies. Pharmacogenomic profiling has shown important results in predicting treatment outcome in cancer biotherapy treated patients $[67,68]$ and has also been considered for chronic rheumatic diseases such as SpA. In CIDs, the absence of common SNPs with a strong effect, and non-genetic sources of patients' heterogeneity suggest that studies with very large numbers of patients would be necessary to obtain a solid correlation of genetic variation with clinical outcome $[69,70]$.

To date, only a few studies have suggested an association between anti-TNF responses in SpA patients and genetic variants, in particular in the TNF and TLR pathways, and downstream NF-kB signaling (Table 1). A number of these variants and genes have also been associated with clinical parameters of remission or low disease in RA (Table 2), supporting the importance of these molecular pathways in the 
mechanisms of action of TNF-blockers. However, the reduced number of studies, the limited size of the cohorts analyzed, and the lack of robust replicated results limit the exploitation of these findings in a clinical setting.

Table 1. Response biomarkers in Spondyloarthritis (SpA).

\begin{tabular}{|c|c|c|c|c|c|}
\hline \multicolumn{6}{|c|}{ GENETIC } \\
\hline $\begin{array}{l}\text { Seitz (2007) } \\
{[71]}\end{array}$ & $\begin{array}{l}\text { AS (22) } \\
\text { RA (54) } \\
\text { PsA (10) }\end{array}$ & $\begin{array}{l}\text { IFX, ADA, } \\
\text { ETN }\end{array}$ & TNF $\alpha-308 \mathrm{G} / \mathrm{G}$ & TNF/TNFR & $\begin{array}{l}\text { Associates with good response } \\
\text { (DAS28 or BASDAl at 24weeks) }\end{array}$ \\
\hline Liu J (2016) [72] & $\begin{array}{l}\text { SpA, IBD, } \\
\text { Ps, PsA. } \\
(1016)\end{array}$ & TNFi & $\begin{array}{l}\text { TNF } \alpha-308 \mathrm{~A} / \mathrm{G} \\
\mathrm{TNF} \alpha-238 \mathrm{~A} / \mathrm{G}\end{array}$ & TNF/TNFR & $\begin{array}{l}\text { G/G predicts good response to TNFi } \\
\text { (ASAS20, ASAS40, BASDAI20,BASDAI50 at } 12 \\
\text { weeks) }\end{array}$ \\
\hline $\begin{array}{l}\text { Fabris M (2016) } \\
\text { [73] }\end{array}$ & SpA (187) & $\begin{array}{l}\text { ADA, IFX, } \\
\text { GOL, ETN }\end{array}$ & $\begin{array}{l}\text { TNF } \alpha-308 \mathrm{~A} / \mathrm{G} \\
\mathrm{IL6}-174 \mathrm{C} / \mathrm{G}\end{array}$ & $\begin{array}{l}\text { TNF/TNFR } \\
\text { IL-6 signaling } \\
\end{array}$ & $\begin{array}{l}\text { G/G predicts survival of the first TNFi } \\
\text { (BASDAl, DAS28-EULAR) }\end{array}$ \\
\hline $\begin{array}{l}\text { Aita A (2018) } \\
\text { [74] }\end{array}$ & SpA (137) & $\begin{array}{l}\text { IFX, ADA, } \\
\text { ETN, GOL }\end{array}$ & TNFRSF1A c.625+10 G & TNF/TNFR & $\begin{array}{l}\text { Associates with late response to TNFi } \\
(\text { BASDAI < 4) }\end{array}$ \\
\hline $\begin{array}{l}\text { Murdaca G } \\
\text { (2014) [75] }\end{array}$ & 57 (PsA) & $\begin{array}{l}\text { ETN } \\
\text { ADA } \\
\text { IFX } \\
\end{array}$ & $\mathrm{TNF} \alpha+489 \mathrm{~A} / \mathrm{G}$ & TNF/TNFR & $\begin{array}{l}\text { A allele associates with the response to ETN. } \\
\text { (PASI, ACR criteria, DAS28, and HAQ at } \\
\text { baseline, } 3 \text { and } 6 \text { months) }\end{array}$ \\
\hline Liu J (2019) [76] & AS (92) & ETN & MYOM2-rs2294066 & & Associates with response (ASASAS40) \\
\hline $\begin{array}{l}\text { Borda (2019) } \\
{[77]}\end{array}$ & SpA (118) & $\begin{array}{l}\text { IFX, ADA, } \\
\text { ETN, GOL }\end{array}$ & $\begin{array}{l}\text { CHUK rs11591741 } \\
\text { MAPKAPK2 rs4240847 } \\
\text { TLR10 rs11096957 } \\
\text { IRAK3 rs11541076 }\end{array}$ & $\begin{array}{l}\text { NFKB } \\
\text { TLR4 } \\
\text { TLR10 } \\
\text { NFKB/TLR4 }\end{array}$ & $\begin{array}{l}\text { Associates with non-response } \\
\text { (BASDAI, BASFI and DAS28-CRP) }\end{array}$ \\
\hline \multicolumn{6}{|c|}{ EPIGENETIC } \\
\hline $\begin{array}{l}\text { Ovejero-Benito } \\
(2018)[78]\end{array}$ & Ps (39) & ADA & $\begin{array}{l}\mathrm{H} 3 \text { and } \mathrm{H} 4 \text { acetylation, } \\
\mathrm{H} 3 \mathrm{~K} 4 \text { and } \mathrm{H} 3 \mathrm{~K} 27 \\
\text { methylation }\end{array}$ & & $\begin{array}{l}\text { Changes in H3K4 were found between Rs } \\
\text { and NR } \\
\text { (PASI75 at } 3 \text { and } 6 \text { months) }\end{array}$ \\
\hline $\begin{array}{l}\text { Ciechomska M } \\
\text { (2018) [79] }\end{array}$ & $\begin{array}{l}\text { AS (13) } \\
\text { RA (10) }\end{array}$ & TNFi & Serum miRNA-5196 & $\begin{array}{l}\text { BCR signaling } \\
\text { MHC-I antigen } \\
\text { processing. }\end{array}$ & $\begin{array}{l}\text { Changes in miRNA- } 5196 \text { can be used as } \\
\text { predictive marker of reduced disease } \\
\text { activity. } \\
\text { Better correlation with changes in DAS20 } \\
\text { and ASDAS than CRP. }\end{array}$ \\
\hline \multicolumn{6}{|c|}{ PROTEINS } \\
\hline $\begin{array}{l}\text { Arends (2011) } \\
\text { [80] }\end{array}$ & AS (92) & ETN & MMP-3 & MMPs & $\begin{array}{l}\text { MMP-3 decreases with TNFi. } \\
\text { Change in MMP-3 serum levels are not } \\
\text { useful for predicting response to ETN. } \\
\text { (ASAS20 and ASA40 at } 3 \text { and } 12 \text { months) }\end{array}$ \\
\hline $\begin{array}{l}\text { Wagner C } \\
(2012)[81]\end{array}$ & AS (100) & GOL & $\begin{array}{l}92 \text { Proteins } \\
\text { profiling }\end{array}$ & $\begin{array}{l}\text { acute } \\
\text { inflammation, } \\
\text { bone } \\
\text { metabolism, } \\
\text { coagulation, } \\
\text { metabolic } \\
\text { factors }\end{array}$ & $\begin{array}{l}\text { Baseline and change in combination of } \\
\text { biomarkers demonstrated stronger } \\
\text { prediction for clinical efficacy than CRP. } \\
\text { (ASAS20 at week 14) }\end{array}$ \\
\hline $\begin{array}{l}\text { Ademowo OS } \\
\text { (2016) [82] }\end{array}$ & PsA (25) & ADA & 57 proteins panel & $\begin{array}{l}\text { Acute } \\
\text { inflammation, } \\
\text { tissue repair, } \\
\text { coagulation }\end{array}$ & $\begin{array}{l}\text { New biomarker panel that can be measured } \\
\text { at baseline to predict PsA patients' response } \\
\text { to biologics. } \\
\text { (DAS28 at } 12 \text { weeks) }\end{array}$ \\
\hline $\begin{array}{l}\text { Turina (2014) } \\
\text { [83] }\end{array}$ & SpA (78) & IFX, ETN & $\begin{array}{l}\text { Calprotectin } \\
\text { MMP3, hsCRP, IL-6, } \\
\text { pentraxin-3, Alpha-2- } \\
\text { macroglobulin, VEGF }\end{array}$ & $\begin{array}{l}\text { Acute phase } \\
\text { reactants } \\
\text { IL-6 signaling } \\
\text { TLR } \\
\text { MMPs }\end{array}$ & $\begin{array}{l}\text { Calprotectin and hs-CRP are good } \\
\text { biomarkers with high sensitivity to change } \\
\text { upon treatment. }\end{array}$ \\
\hline $\begin{array}{l}\text { Østgård RD } \\
\text { (2017) [84] }\end{array}$ & AS (30) & ADA & fecal Calprotectin & TLR & $\begin{array}{l}\text { Elevated baseline fecal calprotectin may } \\
\text { predict better treatment response. } \\
\text { (ASDAS at weeks } 12,20 \text { and 52) }\end{array}$ \\
\hline
\end{tabular}




\begin{tabular}{|c|c|c|c|c|c|}
\hline $\begin{array}{l}\mathrm{HuH}(2019) \\
{[85]}\end{array}$ & AS (262) & $\begin{array}{l}\text { TNFi } \\
\text { NSAID } \\
\text { DMARD }\end{array}$ & Serum Calprotectin & TLR & $\begin{array}{l}\text { Change of calprotectin during first month } \\
\text { could predict patients achieving ASAS } 40 \\
\text { with AUC of } 0.691 .\end{array}$ \\
\hline $\begin{array}{l}\text { Jarlborg M } \\
(2020)[86]\end{array}$ & $\begin{array}{l}\text { AxSpA(451) } \\
\text { RA(969) } \\
\operatorname{PsA}(237)\end{array}$ & TNFi & Serum Calprotectin & TLR & $\begin{array}{l}\text { Calprotectin serum levels associate with } \\
\text { disease activity in RA, SpA but not in PSA. } \\
\text { (SJC, DAS, HAQ, joint radiographs, USPD, } \\
\text { BASDAI, ASDAS, SJC, DAPSA) }\end{array}$ \\
\hline $\begin{array}{l}\text { Baraliakos X } \\
\text { (2019) [87] }\end{array}$ & AS (867) & ETN & C-reactive protein CRP & $\begin{array}{l}\text { IL-6 signaling } \\
\text { Complement } \\
\text { triggering }\end{array}$ & $\begin{array}{l}\text { Very high baseline CRP is a predictor for } \\
\text { week-12 outcomes } \\
\text { (ASAS20, ASAS50, ASDAS-CRP at week 12) }\end{array}$ \\
\hline $\begin{array}{l}\text { Hokstad I } \\
(2019)[88]\end{array}$ & SpA (51) & TNFi & $\begin{array}{l}\text { Complement activation } \\
\text { (sC5b-9 serum levels) }\end{array}$ & $\begin{array}{l}\text { Complement } \\
\text { pathway }\end{array}$ & Decrease from baseline to 6 weeks of TNFi \\
\hline $\begin{array}{l}\text { Chimenti MS } \\
(2012)[89]\end{array}$ & PsA (55) & $\begin{array}{l}\text { ETN } \\
\text { ADA }\end{array}$ & $\begin{array}{l}\text { Plasma complement } \\
\mathrm{C} 3, \mathrm{C} 4 \text {, and } \mathrm{B}\end{array}$ & $\begin{array}{l}\text { Complement } \\
\text { pathway }\end{array}$ & $\begin{array}{l}\text { High baseline C3 levels predict non-response. } \\
\text { (DAS28 and EULAR at baseline and } 22 \text { weeks) }\end{array}$ \\
\hline \multicolumn{6}{|c|}{ TRANSCRIPTS } \\
\hline $\begin{array}{l}\text { Wang XB (2017) } \\
\text { [22] }\end{array}$ & $\begin{array}{l}\text { AS (19) } \\
\text { (PBMC) }\end{array}$ & TNFi & $\begin{array}{l}656 \text { DEG : } \\
\text { CXCR2, } \\
\text { NOTCH1, } \\
\text { TNFRSF1A, } \\
\text { IL6R, } \\
\text { IL1O }\end{array}$ & $\begin{array}{l}\text { Chemokines } \\
\text { NOTCH } \\
\text { signaling } \\
\text { TNF/TNFR } \\
\text { IL-6 signaling } \\
\text { IL-10 }\end{array}$ & $\begin{array}{l}\text { It was not possible to develop a predictive } \\
\text { algorithm for TNFi response } \\
\text { (BASDAI, CRP and ESR) }\end{array}$ \\
\hline $\begin{array}{l}\text { Dolcino M } \\
(2017) \\
{[90]}\end{array}$ & $\begin{array}{l}\text { AS (10) } \\
\text { (PBMC) }\end{array}$ & $\mathrm{ADA}$ & $\begin{array}{l}\text { Assessed } 14500 \text { DEG in } \\
\text { AS patients before and } \\
\text { after ADA treatment }\end{array}$ & $\begin{array}{l}\text { TLR signaling, } \\
\text { TNF signaling, } \\
\text { IFN Type I } \\
\text { Wnt signaling, }\end{array}$ & $\begin{array}{l}\text { ADA treatment modified } 44 \% \text { of DEGs in Rs } \\
\text { compared to } 12 \% \text { in NR. } \\
\text { (BASDAI6) }\end{array}$ \\
\hline \multicolumn{6}{|c|}{ IMMUNE CELL POPULATIONS } \\
\hline $\begin{array}{l}\text { Enginar AU } \\
\text { (2019) [91] }\end{array}$ & $\begin{array}{l}\text { AS (203) } \\
\text { RA (68) }\end{array}$ & TNFi & $\begin{array}{l}\text { NLR } \\
\text { PLR }\end{array}$ & $\begin{array}{l}\text { Neutrophils } \\
\text { Lymphocytes } \\
\text { Platelets }\end{array}$ & $\begin{array}{l}\text { NLR and PLR are strongly correlated with } \\
\text { disease activity, ESR and CRP and decrease } \\
\text { with anti-TNF treatment. } \\
\text { (BASDAI/DAS28 at } 3 \text { and } 6 \text { months) }\end{array}$ \\
\hline $\begin{array}{l}\text { Miyagawa I } \\
\text { (2019) [92] }\end{array}$ & PsA (26) & $\begin{array}{l}\text { ADA } \\
\text { IFX } \\
\text { UST } \\
\text { SEC }\end{array}$ & $\begin{array}{l}\text { Th1 type } \\
\text { Th17 type } \\
\text { Th1/Th17 high } \\
\text { Th1/Th17 low }\end{array}$ & T cells & $\begin{array}{l}\text { bDMARD therapy selected strategically } \\
\text { based on the results of peripheral blood } \\
\text { lymphocyte phenotyping. } \\
\text { (CRP, ESR, SDAl, DAS28 and PASI at baseline } \\
\text { and } 6 \text { months) }\end{array}$ \\
\hline $\begin{array}{l}\text { Schulte-Wrede } \\
\text { U (2018) [93] }\end{array}$ & AS (31) & $\begin{array}{l}\text { ADA, } \\
\text { ETN }\end{array}$ & NK CD8+ cells & NK cells & $\begin{array}{l}\text { Composition of NK cell compartment } \\
\text { predicts therapeutic outcome. } \\
\text { (BASDAI50 between baseline and } 1 \text { to } 6 \\
\text { months) }\end{array}$ \\
\hline $\begin{array}{l}\text { Dulic S(2018) } \\
\text { [94] }\end{array}$ & AS (22) & TNFi & T cell repertoire & T cells & $\begin{array}{l}\text { Increase in CD4HLADR, CD8HLADR and } \\
\text { CD4CD25 cells in Rs. } \\
\text { (ASAS) }\end{array}$ \\
\hline $\begin{array}{l}\text { Xueyi L (2013) } \\
\text { [95] }\end{array}$ & AS (222) & TNFi & Th17 and Treg & T cells & $\begin{array}{l}\text { Higher baseline Th17 in AS vs controls. } \\
\text { Th17 significantly decreased in Rs, increased } \\
\text { in NR. } \\
\text { Treg increased in Rs and decreased in NR. } \\
\text { (ASAS at } 6 \text { months) }\end{array}$ \\
\hline $\begin{array}{l}\text { Andersen T } \\
\text { (2019) [96] }\end{array}$ & $\mathrm{SpA}(30)$ & TNFi & Th17 and Th22 & T cells & 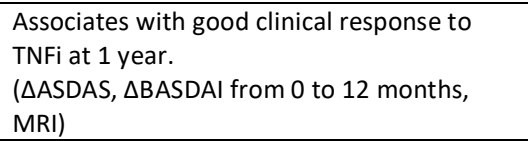 \\
\hline $\begin{array}{l}\text { Yang M (2020) } \\
\text { [97] }\end{array}$ & AS (177) & ETN & $\begin{array}{l}28 \text { T lymphocyte and } \\
12 \text { B lymphocyte } \\
\text { subsets }\end{array}$ & $\begin{array}{l}\text { T cells } \\
\text { B cells }\end{array}$ & $\begin{array}{l}\text { Baseline: imbalance of T and B cell subsets in } \\
\text { AS patients. } \\
\text { After TNFi: decreased naïve CD4+ cells, } \\
\text { increased Treg and B10 cells, } \\
\text { Treg increase positively correlated with CRP } \\
\text { decrease. } \\
\text { (CRP, ASDAS, BASDAl at } 12 \text { weeks) }\end{array}$ \\
\hline
\end{tabular}

Abbreviations: SpA, Spondyloarthritis; AS, Ankylosing Spondylitis; RA, Rheumatoid Arthritis; PsA, Psoriatic Arthritis; IBD, Inflammatory Bowel Disease; ADA, adalimumab; ETN, etanercept; GOL, golimumab; IFX, infliximab; TNFi, UST, ustekinumab; SEC, secukinumab; TNF-inhibitors; HC, healthy controls; Rs, responders; NR, non-responders; MiRNA, Micro RNA; BCR, B cell receptor; NLR, neutrophils/lymphocytes 
ratio; PLR: platelet/lymphocyte ratio, CRP, C-reactive protein; hSCRP, High sensitivity C-reactive protein; SDAI, simplified disease activity index; VEGF, vascular endothelial growth factor; ESR, erythrocyte sedimentation rate; DAS28, disease activity score 28; ASDAS, Ankylosing Spondylitis Disease Activity Score; MMPs, Matrix Metalloproteinases; TLR, Toll like receptor; DEG, differentially expressed genes; SJC, swollen joint count; DAS, Disease Activity Score; HAQ, Health Assessment questionnaire; USPD, ultrasound power Doppler; BASDAI, Bath Ankylosing Spondylitis Disease Activity Index; DAPSA, Disease Activity Index for Psoriatic Arthritis. 
Table 2. Biomarkers in Rheumathoid Arthritis (RA).

\begin{tabular}{|c|c|c|c|c|c|}
\hline \multicolumn{6}{|c|}{ GENETIC } \\
\hline $\begin{array}{l}\text { Pavy S (2010) } \\
\text { [98] }\end{array}$ & $\begin{array}{l}\text { RA (1721, } \\
12 \text { cohorts) } \\
\text { meta- } \\
\text { analysis }\end{array}$ & TNFi & G-308A TNF $\alpha$ & TNF & $\begin{array}{l}\text { No association with treatment response } \\
\text { (deltaDAS28 at } 12 \text { weeks) }\end{array}$ \\
\hline $\begin{array}{l}\text { Zeng Z (2013) } \\
\text { [99] }\end{array}$ & $\begin{array}{l}\text { RA ( } 2127, \\
15 \text { cohorts) } \\
\text { meta- } \\
\text { analysis }\end{array}$ & TNFi & G-308A TNF $\alpha$ & TNF & $\begin{array}{l}\text { Patients with the } G \text { allele respond better } \\
\text { to treatment } \\
\text { (DAS28/ACR) }\end{array}$ \\
\hline \multirow[t]{2}{*}{$\begin{array}{l}\text { Cui J (2013) } \\
{[100]}\end{array}$} & RA (2706) & IFX ADA ETN & \multirow[t]{2}{*}{ rs6427528 CD84 } & \multirow[t]{2}{*}{ SLAM family } & \multirow[t]{2}{*}{$\begin{array}{l}\text { Association with response to ETN } \\
\text { (EULAR criteria at 3-12 months) }\end{array}$} \\
\hline & RA (390) & ETN & & & \\
\hline \multirow[t]{2}{*}{$\begin{array}{l}\text { Swierkot J } \\
\text { (2013) [101] }\end{array}$} & \multirow[t]{2}{*}{ RA (280) } & \multirow[t]{2}{*}{ IFX ADA ETN } & TNFR1A G36A & \multirow[t]{2}{*}{ TNF } & $\begin{array}{l}\text { Associated with remission or low } \\
\text { disease activity (EULAR criteria at } 6 \\
\text { months) }\end{array}$ \\
\hline & & & TNFA C-857T & & $\begin{array}{l}\text { Associated with DAS28 at } 6 \text { months } \\
\text { treatment }\end{array}$ \\
\hline $\begin{array}{l}\text { Ferreiro-Iglesias } \\
\text { A (2016) [102] }\end{array}$ & RA (755) & IFX ADA ETN & $\begin{array}{l}\text { rs10919563 PTPRC } \\
\text { (CD45) } \\
\text { rs1800896 IL10 } \\
\text { rs11591741 CHUK }\end{array}$ & $\begin{array}{l}\text { CD45 } \\
\text { IL10 } \\
\text { NFKB }\end{array}$ & $\begin{array}{l}\text { associated with response to treatment } \\
\text { (EULAR criteria at 3-6 months) }\end{array}$ \\
\hline $\begin{array}{l}\text { Honne K (2016) } \\
{[103]}\end{array}$ & RA (487) & IFX ADA ETN & rs284511 MAP3K7 & TGFbeta, BMP & $\begin{array}{l}\text { associated with treatment response } \\
\text { (deltaDAS28-CRP at 3-6 months) }\end{array}$ \\
\hline \multirow[t]{2}{*}{$\begin{array}{l}\text { Julià A (2016) } \\
\text { [104] }\end{array}$} & RA (372) & \multirow[t]{2}{*}{ IFX ADA ETN } & \multirow[t]{2}{*}{ rs11387825 MED15 } & $\begin{array}{l}\text { PC2, } \\
\text { transcriptional } \\
\text { activation }\end{array}$ & $\begin{array}{l}\text { Association with response to ETN } \\
\text { (EULAR criteria at } 3 \text { months) }\end{array}$ \\
\hline & RA (245) & & & $\begin{array}{l}\text { transcriptional } \\
\text { activation }\end{array}$ & $\begin{array}{l}\text { Association with response to ETN } \\
\text { Association with response to ETN IFX }\end{array}$ \\
\hline $\begin{array}{l}\text { Cui J (2017) } \\
{[105]}\end{array}$ & RA (1094) & IFX ADA ETN & $\begin{array}{l}\text { NFKBIA } \\
\text { AICDA } \\
\text { CDK6 } \\
\end{array}$ & $\begin{array}{l}\text { NFkB, } \\
\text { somatic } \\
\text { hypermutation } \\
\text { class-switch } \\
\text { recombination } \\
\text { cell cycle } \\
\end{array}$ & $\begin{array}{l}\text { No association with response at genome } \\
\text { wide significance. Three genes with } \\
\text { individual } P \text { values of }<0.01 \\
\text { (EULAR criteria at 3-6 months) }\end{array}$ \\
\hline $\begin{array}{l}\text { Marwa OS } \\
(2017)[106]\end{array}$ & $\begin{array}{l}\text { RA (108) } \\
\text { HC (202) }\end{array}$ & ETN IFX & $\begin{array}{l}\text { rs763780 IL17F } 7488 \\
\text { A/G } \\
\text { rs2397084 IL17F } 7383 \\
\text { A/G } \\
\end{array}$ & IL-17 & $\begin{array}{l}\text { Association with response. } \\
\text { Serum levels of IL-17 higher in RA versus } \\
\text { HC } \\
\text { (DAS28) }\end{array}$ \\
\hline $\begin{array}{l}\text { Sieberts SK } \\
\text { (2016) [107] }\end{array}$ & $\begin{array}{l}\text { RA ( } 2706 \\
\text { training) } \\
\text { RA (591 } \\
\text { validation) }\end{array}$ & TNFi & $\begin{array}{l}\text { Published SNP data } \\
\text { predicting response to } \\
\text { TNFi } \\
\text { Meta-analysis } \\
\end{array}$ & & $\begin{array}{l}\text { no significant genetic contribution to } \\
\text { prediction accuracy of response } \\
\text { (deltaDAS28 at 3-12 months) }\end{array}$ \\
\hline \multirow{2}{*}{$\begin{array}{l}\text { Cherlin S (2018) } \\
{[108]}\end{array}$} & RA (1819) & TNFi & \multirow{2}{*}{$\begin{array}{l}\text { GWAS data from } \\
\text { Matura consortium }\end{array}$} & & \multirow{2}{*}{$\begin{array}{l}\text { poor prediction of response using } 11 \\
\text { different statistical methods } \\
\text { (delta CRP, delta ESR, delta SJC28 } \\
\text { at 3-6 months) }\end{array}$} \\
\hline & RA (657) & MTX & & & \\
\hline $\begin{array}{l}\text { Guan (2019) } \\
{[109]}\end{array}$ & $\begin{array}{l}\text { RA (2706 } \\
\text { training) } \\
\text { RA (591 } \\
\text { validation) }\end{array}$ & TNFi & $\begin{array}{l}\text { rs1990099 MAGI2 } \\
\text { rs10833455 NELL1 } \\
\text { rs10833456 NELL1 }\end{array}$ & $\begin{array}{l}\text { small GTPase } \\
\text { signalling } \\
\text { osteochondro- } \\
\text { genesis }\end{array}$ & $\begin{array}{l}\text { clinical and SNP data modelled to } \\
\text { predict treatment response. Genetic } \\
\text { information only marginally improves } \\
\text { the prediction } \\
\text { (deltaDAS28 at 3-12 months) }\end{array}$ \\
\hline $\begin{array}{l}\text { Ferreiro-Iglesias } \\
\text { A (2019) [110] }\end{array}$ & RA (3978, & IFX ETN ADA & rs2378945 NUBPL & $\begin{array}{l}\text { NADH } \\
\text { dehydrogenase }\end{array}$ & $\begin{array}{l}\text { association with response to ETN } \\
\text { (EULAR criteria at } 3 \text { months) }\end{array}$ \\
\hline
\end{tabular}




\begin{tabular}{|c|c|c|c|c|c|}
\hline & $\begin{array}{l}755 \\
\text { replication) }\end{array}$ & & $\begin{array}{l}\text { rs12142623 } \\
\text { rs4651370 PLA2G4A }\end{array}$ & $\begin{array}{l}\text { arachidonic } \\
\text { acid pathway }\end{array}$ & \\
\hline $\begin{array}{l}\text { Bai M (2019) } \\
{[111]}\end{array}$ & $\begin{array}{l}\text { RA (507) } \\
\text { HC (499) }\end{array}$ & & $\begin{array}{l}\text { rs969129 IL7R } \\
\text { rs6451231 IL7R }\end{array}$ & IL-7 & Association with RA risk \\
\hline \multicolumn{6}{|c|}{ EPIGENETIC } \\
\hline $\begin{array}{l}\text { Castro-Villegas C } \\
(2015)[112]\end{array}$ & RA (95) & IFX ADA ETN & $\begin{array}{l}\text { Serum microRNAs } \\
\text { signature (miR-23 and } \\
\text { miR-223) }\end{array}$ & $\begin{array}{l}\text { Putative } \\
\text { targets: CHUK, } \\
\text { IL6R, IRAK2, } \\
\text { BMPR2 }\end{array}$ & $\begin{array}{l}\text { miR-23 and miR-223 baseline levels } \\
\text { correlate with treatment response } \\
\text { (EULAR criteria at } 6 \text { months) }\end{array}$ \\
\hline $\begin{array}{l}\text { Krintel SB (2015) } \\
{[113]}\end{array}$ & RA (180) & ADA & $\begin{array}{l}\text { microRNA in pre- } \\
\text { treatment whole blood }\end{array}$ & $\begin{array}{l}\text { ER alpha } \\
\text { extracellular } \\
\text { matrix (Cyr61) } \\
\text { Chemokines } \\
\text { (CXCL12) }\end{array}$ & $\begin{array}{l}\text { low expression of miR-22 and high } \\
\text { expression of miR- } 886.3 p \text { is associated } \\
\text { with response (EULAR criteria at } 3 \\
\text { months) }\end{array}$ \\
\hline \multicolumn{6}{|c|}{ TRANSCRIPTS } \\
\hline $\begin{array}{l}\text { Oswald M (2015) } \\
{[114]}\end{array}$ & RA (240) & $\begin{array}{l}\text { IFX ADA ETN } \\
\text { GOL CTZ }\end{array}$ & $\begin{array}{l}\text { gene modules for } \\
\text { plasmacells, } \\
\text { B cells, MHC/ribosome } \\
\text { proteins, T cells }\end{array}$ & $\begin{array}{l}\text { B cells } \\
\text { T cells }\end{array}$ & $\begin{array}{l}\text { increased expression after TNFi in } \\
\text { responders. No baseline difference } \\
\text { between Rs and NRs } \\
\text { (EULAR criteria at } 14 \text { weeks) }\end{array}$ \\
\hline $\begin{array}{l}\text { Huang Q-I (2017) } \\
{[115]}\end{array}$ & $\begin{array}{l}\text { RA (384, } \\
8 \text { cohorts) } \\
\text { meta- } \\
\text { analysis }\end{array}$ & IFX & $\begin{array}{l}\text { FKBP1A } \\
\text { FGF12 } \\
\text { ANO1 } \\
\text { LRRC31 } \\
\text { AKR1D1 }\end{array}$ & $\begin{array}{l}\text { regulation of } \\
\text { trans- } \\
\text { membrane } \\
\text { transport }\end{array}$ & $\begin{array}{l}\text { Logistic regression model of the } 5 \text { genes } \\
\text { predicts IFX response. } \\
\text { (EULAR/ACR criteria at week } 6,14,22 \text { ) }\end{array}$ \\
\hline $\begin{array}{l}\text { Byng-Maddick R } \\
\text { (2017) [116] }\end{array}$ & $\begin{array}{l}\text { RA (37) } \\
\text { HC (13) }\end{array}$ & IFX ETN ADA & TNF-inducible genes & TNF & $\begin{array}{l}\text { TNFi do not affect TNF-inducible gene } \\
\text { expression at the site of acute immune } \\
\text { challenge (tuberculin test). TNF- } \\
\text { dependent gene expression decreased } \\
\text { in blood sample from treated patients }\end{array}$ \\
\hline \multicolumn{6}{|c|}{ PROTEIN } \\
\hline $\begin{array}{l}\text { Shi R (2018) } \\
\text { [117] }\end{array}$ & RA (69) & ETN & $\begin{array}{l}\text { IL-6 } \\
\text { survivin }\end{array}$ & $\begin{array}{l}\text { IL-6 } \\
\text { apoptosis }\end{array}$ & $\begin{array}{l}\text { Baseline serum IL-6 increased in Rs to } \\
\text { ETN, baseline survivin decreased. Only } \\
\text { survivin and CRP are independent } \\
\text { predictive factors }\end{array}$ \\
\hline $\begin{array}{l}\text { BK Han (2016) } \\
\text { [118] }\end{array}$ & RA (29) & TNFi & $\begin{array}{l}\text { Serum CXCL10 and } \\
\text { CXCL13 }\end{array}$ & chemokines & $\begin{array}{l}\text { Rs have higher baseline levels of CXCL10 } \\
\text { and CXCL13 compared to NRs } \\
\text { (EULAR criteria at week 14) }\end{array}$ \\
\hline $\begin{array}{l}\text { Haschka J (2016) } \\
\text { [119] }\end{array}$ & $\begin{array}{l}\text { RA in } \\
\text { DAS28 } \\
\text { remission } \\
(101)\end{array}$ & $\begin{array}{l}\text { conventional } \\
\text { and/or } \\
\text { biological } \\
\text { DMARD, } \\
\text { randomized to } \\
\text { tapering }\end{array}$ & $\begin{array}{l}\text { anticitrullinated } \\
\text { protein antibodies } \\
\text { (ACPA) }\end{array}$ & & $\begin{array}{l}\text { ACPA positivity independently } \\
\text { associated with relapse }\end{array}$ \\
\hline $\begin{array}{l}\text { Curtis JR (2012) } \\
\text { [120] }\end{array}$ & $\begin{array}{l}\text { RA (230) } \\
\text { RA (45) } \\
\text { (treatment } \\
\text { response) }\end{array}$ & $\begin{array}{l}\text { MTX } \\
\text { anti-TNFa }\end{array}$ & $\begin{array}{l}\text { Multi-biomarker } \\
\text { disease activity } \\
\text { (MBDA) score : EGF, } \\
\text { VEGF-A, leptin, IL-6, } \\
\text { SAA, CRP, VCAM-1, } \\
\text { MMP-1, MMP-3, } \\
\text { TNFRI, YKL-40, and } \\
\text { resistin }\end{array}$ & $\begin{array}{l}\text { EGF } \\
\text { TNF } \\
\text { IL-6 } \\
\text { VEGF } \\
\text { Integrins } \\
\text { MMPs } \\
\text { Apolipo- } \\
\text { proteins }\end{array}$ & $\begin{array}{l}\text { significant association with DAS28-CRP. } \\
\text { Changes in MBDA score discriminates } \\
\text { clinical Rs from NRs } \\
\text { (ACR20 week 6-12, deltaDAS28-CRP } \\
\text { week 6-12) }\end{array}$ \\
\hline $\begin{array}{l}\text { Centola M } \\
(2013)[121]\end{array}$ & $\begin{array}{l}\text { RA ( } 676, \\
5 \text { cohorts) }\end{array}$ & $\begin{array}{l}\text { Conventional } \\
\text { and biological } \\
\text { DMARDs }\end{array}$ & MBDA & $\begin{array}{l}\text { MBDA } \\
\text { pathways }\end{array}$ & $\begin{array}{l}\text { Discriminates patients with low vs. } \\
\text { moderate/high clinical disease activity. } \\
\text { Tracks changes in DAS28-CRP }\end{array}$ \\
\hline $\begin{array}{l}\text { Hirata S (2015) } \\
\text { [122] }\end{array}$ & RA (84) & ADA ETN IFX & MBDA & $\begin{array}{l}\text { MBDA } \\
\text { pathways }\end{array}$ & $\begin{array}{l}\text { MBDA scores correlate with disease } \\
\text { activity. } \\
\text { Tracks response to treatment } \\
\text { (deltaDAS28-CRP, deltaDAS28-ESR, at } 24 \\
\text { and } 52 \text { weeks) }\end{array}$ \\
\hline $\begin{array}{l}\text { Hirata S (2016) } \\
\text { [123] }\end{array}$ & RA (83) & ADA ETN IFX & MBDA & $\begin{array}{l}\text { MBDA } \\
\text { pathways }\end{array}$ & $\begin{array}{l}\text { MBDA score and DAS28 at week } 24 \text { are } \\
\text { significant predictors of radiographic } \\
\text { progression at week } 52\end{array}$ \\
\hline $\begin{array}{l}\text { Li W (2016) } \\
{[124]}\end{array}$ & RA (163) & & MBDA & $\begin{array}{l}\text { MBDA } \\
\text { pathways }\end{array}$ & $\begin{array}{l}\text { MBDA score is independently associated } \\
\text { with radiographic progression. }\end{array}$ \\
\hline
\end{tabular}




\begin{tabular}{|c|c|c|c|c|c|}
\hline & & & & & $\begin{array}{l}\text { Significantly discriminates patients with } \\
\text { low CRP and high risk of progression. }\end{array}$ \\
\hline $\begin{array}{l}\text { Fleischmann R } \\
\text { (2016) [125] }\end{array}$ & $\begin{array}{l}\text { RA (241) } \\
\text { RA (251) }\end{array}$ & $\begin{array}{l}\mathrm{ABC} \\
\mathrm{ADA}\end{array}$ & MBDA & $\begin{array}{l}\text { MBDA } \\
\text { pathways }\end{array}$ & $\begin{array}{l}\text { MBDA does not reflect the status of } \\
\text { radiographic progression, nor disease } \\
\text { activity }\end{array}$ \\
\hline $\begin{array}{l}\text { Hambardzumyan } \\
\text { K (2016) [126] }\end{array}$ & RA (205) & $\begin{array}{l}\text { MTX+IFX, } \\
\text { conventional } \\
\text { DMARDs }\end{array}$ & MBDA & $\begin{array}{l}\text { MBDA } \\
\text { pathways }\end{array}$ & $\begin{array}{l}\text { MBDA score at baseline, month } 3 \text { and } \\
\text { year } 1 \text { is correlated with subsequent } \\
\text { rapid radiographic progression }\end{array}$ \\
\hline $\begin{array}{l}\text { Rech J (2016) } \\
{[127]}\end{array}$ & RA (94) & $\begin{array}{l}\text { conventional } \\
\text { and/or } \\
\text { biological } \\
\text { DMARD } \\
\text { (tapering) } \\
\end{array}$ & MBDA & $\begin{array}{l}\text { MBDA } \\
\text { pathways }\end{array}$ & $\begin{array}{l}\text { Higher baseline MBDA scores } \\
\text { significantly associated with relapse. } \\
\text { ACPA positivity and MBDA are } \\
\text { independent predictors of relapse. }\end{array}$ \\
\hline $\begin{array}{l}\text { Hambardzumyan } \\
\text { K (2017) [128] }\end{array}$ & RA (157) & $\begin{array}{l}\text { MTX+IFX, } \\
\text { conventional } \\
\text { DMARDs }\end{array}$ & MBDA & $\begin{array}{l}\text { MBDA } \\
\text { pathways }\end{array}$ & $\begin{array}{l}\text { MBDA score is associated with response } \\
\text { to second-line therapy and with } \\
\text { response to IFX } \\
\text { (EULAR criteria at } 3 \text { and } 12 \text { months) }\end{array}$ \\
\hline $\begin{array}{l}\text { Krabbe S (2017) } \\
{[129]}\end{array}$ & RA (52) & ADA & MBDA & $\begin{array}{l}\text { MBDA } \\
\text { pathways }\end{array}$ & $\begin{array}{l}\text { High MBDA in patients with structural } \\
\text { progression }\end{array}$ \\
\hline $\begin{array}{l}\text { Bouman CAM } \\
\text { (2017) [130] }\end{array}$ & RA (171) & $\begin{array}{l}\text { ADA ETN } \\
\text { randomized } \\
\text { discontinuation }\end{array}$ & MBDA & $\begin{array}{l}\text { MBDA } \\
\text { pathways }\end{array}$ & $\begin{array}{l}\text { Baseline MBDA score is not predictive } \\
\text { for clinical outcome in the taper group, } \\
\text { but predicts major flares in the usual } \\
\text { care group. Radiographic progression } \\
\text { was minimal and not predicted by } \\
\text { MDBA score. }\end{array}$ \\
\hline $\begin{array}{l}\text { Ghiti-Moghadam } \\
\text { M (2018) [131] }\end{array}$ & $\begin{array}{l}\text { RA (439) } \\
\text { Low disease } \\
\text { activity }\end{array}$ & $\begin{array}{l}\text { TNFi } \\
\text { randomized } \\
\text { discontinuation }\end{array}$ & MBDA & $\begin{array}{l}\text { MBDA } \\
\text { pathways }\end{array}$ & $\begin{array}{l}\text { Low MDBA score independently } \\
\text { associated with successful } \\
\text { discontinuation }\end{array}$ \\
\hline $\begin{array}{l}\text { Bechman K } \\
(2018) \\
{[132]}\end{array}$ & RA (152) & $\begin{array}{l}\text { conventional } \\
\text { and/or } \\
\text { biological } \\
\text { DMARD }\end{array}$ & MBDA & $\begin{array}{l}\text { MBDA } \\
\text { pathways }\end{array}$ & $\begin{array}{l}\text { Baseline MBDA score not predictive of } \\
\text { flares }\end{array}$ \\
\hline $\begin{array}{l}\text { Curtis JR (2019) } \\
{[133]}\end{array}$ & $\begin{array}{l}\text { RA ( } 929, \\
5 \text { cohorts) } \\
\text { Meta- } \\
\text { analysis } \\
\end{array}$ & $\begin{array}{l}\text { MTX, ADA, IFX, } \\
\text { ABA }\end{array}$ & MBDA & $\begin{array}{l}\text { MBDA } \\
\text { pathways }\end{array}$ & $\begin{array}{l}\text { High negative predictive value of MBDA } \\
\text { score for radiographic progression }\end{array}$ \\
\hline $\begin{array}{l}\text { Brahe CH (2019) } \\
\text { [133] }\end{array}$ & $\begin{array}{l}\text { early RA } \\
(180)\end{array}$ & $\begin{array}{l}\text { MTX } \\
\text { MTX+ADA }\end{array}$ & MBDA & $\begin{array}{l}\text { MBDA } \\
\text { pathways }\end{array}$ & $\begin{array}{l}\text { Baseline MBDA was associated with } \\
\text { radiographic progression at } 1 \text { year, delta } \\
\text { MBDA (baseline to } 3 \text { months) with } \\
\text { clinical remission at } 6 \text { months } \\
\text { (DAS28-CRP } \leq 2.6 \text { ) }\end{array}$ \\
\hline $\begin{array}{l}\text { Cuppen B (2018) } \\
{[134]}\end{array}$ & $\begin{array}{l}\text { RA (65 } \\
\text { training) } \\
\text { RA (185 } \\
\text { validation) }\end{array}$ & $\begin{array}{l}\text { IFX ADA ETN } \\
\text { GOL CTZ }\end{array}$ & $\begin{array}{l}\text { CCL3, CCL17, CCL19, } \\
\text { CCL22, IL-4, IL-6, IL-7, } \\
\text { IL-15, SCD14, sCD74, } \\
\text { sIL-1R1, sTNFRII }\end{array}$ & $\begin{array}{l}\text { Chemokines } \\
\text { IL6 } \\
\text { IL1 } \\
\text { TNF }\end{array}$ & $\begin{array}{l}\text { The protein score marginally improves } \\
\text { prediction of DAS } 28 \text { at } 3 \text { months after } \\
\text { treatment, compared to clinical scores }\end{array}$ \\
\hline $\begin{array}{l}\text { Choi IY (2015) } \\
\text { [135] }\end{array}$ & RA (170) & ADA IFX RIX & $\begin{array}{l}\text { Serum calprotectin } \\
\text { (MRP8/14, S100A8/A9) }\end{array}$ & TLR & $\begin{array}{l}\text { Baseline calprotectin serum levels are } \\
\text { higher in responders. Levels in Rs } \\
\text { decrease after treatment } \\
\text { (EULAR criteria at } 16 \text { weeks) }\end{array}$ \\
\hline $\begin{array}{l}\text { Nair SC (2016) } \\
{[136]}\end{array}$ & RA (170) & ADA IFX RIX & $\begin{array}{l}\text { Serum calprotectin } \\
\text { (MRP8/14, S100A8/A9) }\end{array}$ & TLR & $\begin{array}{l}\text { Logistic regression model of baseline } \\
\text { calprotectin serum levels predicts } \\
\text { response } \\
\text { (EULAR criteria at } 16 \text { weeks) }\end{array}$ \\
\hline $\begin{array}{l}\text { Nordal HH } \\
(2016)[137]\end{array}$ & RA (39) & IFX & $\begin{array}{l}\text { Serum calprotectin } \\
\text { (MRP8/14, S100A8/A9) }\end{array}$ & TLR & $\begin{array}{l}\text { Weak association of baseline } \\
\text { calprotectin levels with radiographic } \\
\text { progression. No predictive power for } \\
\text { outcome. }\end{array}$ \\
\hline $\begin{array}{l}\text { Smith SL (2017) } \\
\text { [138] }\end{array}$ & RA (236) & ETN & Serum S100A9 & TLR & $\begin{array}{l}\text { No association with response, nor } \\
\text { clinical parameters of disease activity } \\
\text { (EULAR criteria at } 6 \text { months) }\end{array}$ \\
\hline $\begin{array}{l}\text { Inciarte-Mundo J } \\
\text { (2018) [139] }\end{array}$ & $\begin{array}{l}\text { RA (47) } \\
\text { PsA (56) }\end{array}$ & IFX ADA ETN & $\begin{array}{l}\text { Serum calprotectin } \\
\text { (MRP8/14, S100A8/A9) }\end{array}$ & TLR & $\begin{array}{l}\text { Baseline calprotectin serum } \\
\text { independently predicts relapse }\end{array}$ \\
\hline
\end{tabular}




\begin{tabular}{|c|c|c|c|c|c|}
\hline \multirow[t]{2}{*}{$\begin{array}{l}\text { Tweehuysen L } \\
\text { (2018) [140] }\end{array}$} & RA (125) & $\begin{array}{l}\text { ADA ETN } \\
\text { starting }\end{array}$ & \multirow[t]{2}{*}{$\begin{array}{l}\text { Serum calprotectin } \\
\text { (MRP8/14, S100A8/A9) }\end{array}$} & \multirow[t]{2}{*}{ TLR } & \multirow{2}{*}{$\begin{array}{l}\text { Baseline calprotectin levels predictive of } \\
\text { response only in one cohort. No } \\
\text { predictive advantage over clinical } \\
\text { factors } \\
\text { (EULAR criteria at } 6 \text { months) }\end{array}$} \\
\hline & RA (102) & tapering & & & \\
\hline $\begin{array}{l}\text { Yunchun L } \\
\text { (2018) [141] }\end{array}$ & RA (180) & rhTNFR-Fc & $\begin{array}{l}\text { Serum calprotectin } \\
\text { (MRP8/14, S100A8/A9) }\end{array}$ & TLR & $\begin{array}{l}\text { Rapid decrease of calprotectin levels is } \\
\text { associated with a positive clinical } \\
\text { response (ACR20). Levels decrease } \\
\text { during treatment. }\end{array}$ \\
\hline $\begin{array}{l}\text { De Moel EC } \\
\text { (2019) [142] }\end{array}$ & $\begin{array}{l}\text { RA (104) } \\
\text { RA (57) }\end{array}$ & $\begin{array}{l}\text { tapering } \\
\text { DMARDs or } \\
\text { biologics }\end{array}$ & $\begin{array}{l}\text { Serum calprotectin } \\
\text { (MRP8/14, S100A8/A9) }\end{array}$ & TLR & $\begin{array}{l}\text { Higher calprotectin is associated with } \\
\text { increased risk of relapse after TNFi } \\
\text { tapering }\end{array}$ \\
\hline \multicolumn{6}{|c|}{ IMMUNE CELL POPULATIONS } \\
\hline $\begin{array}{l}\text { Daien Cl (2014) } \\
\text { [143] }\end{array}$ & $\begin{array}{l}\text { RA (96) } \\
\text { HC (31) }\end{array}$ & TNFi & $\begin{array}{l}\text { memory B cells in } \\
\text { peripheral blood }\end{array}$ & B cells & $\begin{array}{l}\text { CD27+ cells produced three times more } \\
\text { TNF } \alpha \text { than did naïve B cells. } \\
\text { Higher proportion of CD27+ memory B } \\
\text { cells at baseline is associated with } \\
\text { response to TNFi } \\
\text { (EULAR criteria at } 3 \text { months) }\end{array}$ \\
\hline $\begin{array}{l}\text { Citro A (2015) } \\
{[144]}\end{array}$ & RA (16) & ETN & CD8+ T cells & CD8+ T cells & $\begin{array}{l}\text { Percentage apoptotic epitope-specific } \\
\text { effector } C D 8^{+} T \text { cells is more elevated in } \\
\text { Rs } \\
\text { (EULAR criteria at } 6 \text { months) }\end{array}$ \\
\hline $\begin{array}{l}\text { Hull DN (2016) } \\
{[145]}\end{array}$ & RA (25) & ADA ETN & Th17 cells at baseline & Th17 & $\begin{array}{l}\text { Higher frequencies of baseline Th17 } \\
\text { cells is associated with worse response } \\
\text { defined by ultrasonography (synovitis). } \\
\text { Th17 frequency increases with TNFi } \\
\text { therapy }\end{array}$ \\
\hline $\begin{array}{l}\text { Salomon S } \\
\text { (2017) [146] }\end{array}$ & $\begin{array}{l}\text { RA (31) } \\
\text { HC (17) }\end{array}$ & TNFi ABT TCZ & $\begin{array}{l}\text { CD24hiCD27+ Breg } \\
\text { Th17 cells }\end{array}$ & $\begin{array}{l}\text { B cells } \\
\text { Th17 }\end{array}$ & $\begin{array}{l}\text { Higher levels of Breg at baseline is } \\
\text { associated with DAS28 remission at } 6 \\
\text { months (ABT) } \\
\text { Lower baseline Th17 is associated with a } \\
\text { good response at } 6 \text { months. } \\
\text { RA have reduced Breg, Th17 }\end{array}$ \\
\hline $\begin{array}{l}\text { Khanniche A } \\
\text { (2018) [34] }\end{array}$ & $\begin{array}{l}\text { RA (49) } \\
\text { HC (65) }\end{array}$ & GOL, MTX & $\begin{array}{l}\text { Increased effector } \\
\text { memory and } \\
\text { decreased central } \\
\text { memory CD8+ and } \\
\text { CD4+ T cells after TNFi }\end{array}$ & $\begin{array}{l}\text { CD8, CD4 T } \\
\text { cells }\end{array}$ & $\begin{array}{l}\text { Increased effector CD8+ T cells } \\
\text { responses to viral antigens in GOL } \\
\text { treated patients }\end{array}$ \\
\hline $\begin{array}{l}\text { Lee HN (2019) } \\
\text { [147] }\end{array}$ & $\begin{array}{l}\text { RA (82) } \\
\text { HC (328) }\end{array}$ & ADA ETN IFX & $\begin{array}{l}\text { Neutrophil/ } \\
\text { lymphocyte ratio (NLR) } \\
\text { Platelet/ } \\
\text { lymphocyte ratio (PLR) }\end{array}$ & $\begin{array}{l}\text { Neutrophils } \\
\text { Lymphocytes } \\
\text { platelets }\end{array}$ & $\begin{array}{l}\text { High NLR and PLR at baseline correlated } \\
\text { with treatment response at } 12 \text {, but not } \\
\text { at } 24 \text { weeks } \\
\text { (EULAR criteria, DAS28-ESR) }\end{array}$ \\
\hline $\begin{array}{l}\text { Cianciotti BC } \\
(2020)[148]\end{array}$ & $\begin{array}{l}\text { RA (27) } \\
\text { HC (20) }\end{array}$ & ETN & CD45RA+CD62L+CD96+ & $\begin{array}{l}\text { Tscm } \\
\text { IL-17 }\end{array}$ & $\begin{array}{l}\text { Expansion of citrullinated vimentin } \\
\text { specific Tscm in RA, reduction after } \\
\text { TNFi. Expansion of Th17 and IL-17+ } \\
\text { Tscm in RA, reduction after TNFi }\end{array}$ \\
\hline $\begin{array}{l}\text { Rodriguez- } \\
\text { Martin E (2020) } \\
{[149]}\end{array}$ & RA (98) & $\begin{array}{l}\text { TNFi } \\
\text { MTX, PREDN }\end{array}$ & CD19+CD27- & Naïve B cells & $\begin{array}{l}\text { Modest positive correlation of } \mathrm{B} / \mathrm{T} \text { cell } \\
\text { ratio with clinical outcome } \\
\text { (DAS28 } \leq 2.6 \text { at } 6 \text { months) }\end{array}$ \\
\hline \multicolumn{6}{|c|}{ MULTIDIMENSIONAL PARAMETERS } \\
\hline \multicolumn{6}{|c|}{ IMMUNE CELLS and PROTEINS } \\
\hline $\begin{array}{l}\text { Bystrom J (2017) } \\
\text { [150] }\end{array}$ & RA (97) & $\begin{array}{l}\text { ADA ETN GOL } \\
\text { CTZ, MTX }\end{array}$ & GM-CSF, IL-1beta & GM-CSF, IL1 & $\begin{array}{l}\text { GM-CSF mostly produced by T cells. GM- } \\
\text { CSF+ T cells are TNF } \alpha \text { positive and IL-17 } \\
\text { negative. High pre-treatment blood } \\
\text { levels of GM-CSF are predictive of } \\
\text { response to TNFi (EULAR criteria at } 3 \\
\text { months) }\end{array}$ \\
\hline \multicolumn{6}{|c|}{ IMMUNE CELLS and TRANSCRIPTS } \\
\hline $\begin{array}{l}\text { Lewis MJ (2019) } \\
\text { [26] }\end{array}$ & RA (90) & $\begin{array}{l}\text { Conventional } \\
\text { DMARDs }\end{array}$ & $\begin{array}{l}\text { Synovial pathotypes } \\
\text { determined by } \\
\text { immunohisto- }\end{array}$ & $\begin{array}{l}\text { Type I IFN } \\
\text { signature, } \\
\text { chemokine } \\
\text { modules, }\end{array}$ & $\begin{array}{l}\text { Synovial gene modules correlate with } \\
\text { DAS28-CRP, and with delta DAS28-CRP } \\
\text { at } 6 \text { months }\end{array}$ \\
\hline
\end{tabular}




\begin{tabular}{|c|c|c|c|c|c|}
\hline & & & $\begin{array}{l}\text { chemistry and synovial } \\
\text { gene expression }\end{array}$ & $\begin{array}{l}\text { monocyte, DC } \\
\text { and B cell } \\
\text { modules }\end{array}$ & \\
\hline $\begin{array}{l}\text { Lliso-Ribera G } \\
\text { (2019) [151] }\end{array}$ & $\begin{array}{l}\text { early RA } \\
(200)\end{array}$ & $\begin{array}{l}\text { Conventional } \\
\text { DMARDs }\end{array}$ & $\begin{array}{l}\text { Synovial pathotypes } \\
\text { determined by } \\
\text { immunohisto- } \\
\text { chemistry and synovial } \\
\text { gene expression }\end{array}$ & $\begin{array}{l}\text { Neutrophils } \\
\text { lymphocytes }\end{array}$ & $\begin{array}{l}\text { Baseline lympho-myeloid pathotype is } \\
\text { significantly associated with } \\
\text { requirement for biologics at } 12 \text { months. } \\
\text { These patients have higher expression } \\
\text { of genes regulating B and T cell } \\
\text { differentiation/ activation, MMP1, } \\
\text { TIMP1, TNFA }\end{array}$ \\
\hline \multicolumn{6}{|c|}{ GENETIC and TRANSCRIPTS } \\
\hline $\begin{array}{l}\text { Aterido A (2019) } \\
\text { [56] }\end{array}$ & $\begin{array}{l}\text { RA (11) } \\
\text { RA (384 } \\
\text { exploration) } \\
\text { RA (2706 } \\
\text { validation) } \\
\end{array}$ & IFX ADA ETN & $\begin{array}{l}\text { synovial gene } \\
\text { coexpression module } \\
\text { (18 genes) }\end{array}$ & $\begin{array}{l}\text { Nucleotide } \\
\text { metabolism } \\
\text { IL-7 }\end{array}$ & $\begin{array}{l}\text { The gene coexpression module is } \\
\text { associated with response to ADA } \\
\text { (EULAR criteria at week 14) }\end{array}$ \\
\hline $\begin{array}{l}\text { Cherlin S (2020) } \\
\text { [60] }\end{array}$ & $\begin{array}{l}\text { RA (4741) } \\
\text { RA (90 } \\
\text { replication) }\end{array}$ & $\begin{array}{l}\text { IFX ETN ADA } \\
\text { GOL CTZ }\end{array}$ & IL18RAP & IL-18 & $\begin{array}{l}\text { Transcript levels in blood correlate with } \\
\text { ESR changes after treatment }\end{array}$ \\
\hline \multicolumn{6}{|c|}{ GENETIC and EPIGENETIC } \\
\hline $\begin{array}{l}\text { Massey J (2018) } \\
{[152]}\end{array}$ & RA (1752) & IFX ADA ETN & $\begin{array}{l}\text { rs7195994 FTO } \\
\text { rs2540767 } \\
\text { rs11599217 DOCK1 } \\
\text { rs10739537 BRINP1 } \\
\text { rs948138 } \\
\text { MMP20/MMP27 } \\
\text { rs2187874 } \\
\text { ZNF595,ZNF718 }\end{array}$ & $\begin{array}{l}\text { Phagocytosis, } \\
\text { cell migration, } \\
\text { FoxP3, Treg } \\
\text { MMPs }\end{array}$ & $\begin{array}{l}\text { Association with response to TNFi } \\
\text { (delta DAS28-ESR at 3-6 months) }\end{array}$ \\
\hline \multicolumn{6}{|c|}{ TRANSCRIPT and PROTEIN } \\
\hline \multirow[t]{2}{*}{$\begin{array}{l}\text { Farutin V (2019) } \\
\text { [153] }\end{array}$} & $\begin{array}{l}\text { RA (40) } \\
\text { RA (36) }\end{array}$ & ADA IFX & $\begin{array}{l}\text { increased innate cell } \\
\text { signature at baseline in } \\
\text { Rs, increased adaptive } \\
\text { cell signature in NRs }\end{array}$ & Myeloid cells & \multirow{2}{*}{$\begin{array}{l}\text { Downregulation of myeloid and } \\
\text { platelets genes, upregulation of T and B } \\
\text { cell markers after TNFi. } \\
\text { Increased NLR at baseline correlates } \\
\text { with increased probability of response } \\
\text { (EULAR criteria at } 3 \text { months) }\end{array}$} \\
\hline & RA (1962) & & NLR & & \\
\hline \multicolumn{6}{|c|}{ TRANSCRIPTS and EPIGENETICS } \\
\hline $\begin{array}{l}\text { Tao W (2020) } \\
{[154]}\end{array}$ & RA (89) & ADA, ETN & $\begin{array}{l}\text { Gene expression and } \\
\text { DNA methylation } \\
\text { profiling }\end{array}$ & & $\begin{array}{l}\text { Random Forest model based on } \\
\text { differentially expressed genes or DNA } \\
\text { methylation predicts response to ADA or } \\
\text { ETN (DAS28 at } 6 \text { months) }\end{array}$ \\
\hline \multicolumn{6}{|c|}{ GENETIC, EPIGENETICS, TRANSCRIPTS } \\
\hline $\begin{array}{l}\text { Spiliopoulou A } \\
\text { (2019) [155] }\end{array}$ & RA (2938) & ADA ETN CTZ & CD39 CD40 & $\begin{array}{l}\text { CD39/CD73 } \\
\text { pathway } \\
\text { T cell } \\
\text { costimulation }\end{array}$ & $\begin{array}{l}\text { RA risk score at the CD40 locus, and } \\
\text { expression score for CD39 on CD4 T cells } \\
\text { are associated with response to TNFi } \\
\text { (delta SJC, delta ESR at } 6 \text { months) }\end{array}$ \\
\hline \multicolumn{6}{|c|}{ GENETIC , TRANSCRIPTS, PROTEINS } \\
\hline $\begin{array}{l}\text { Folkersen L } \\
(2016)[156]\end{array}$ & $\begin{array}{l}\text { RA (185, of } \\
\text { which } 59 \\
\text { with TNFi) } \\
\text { HC (61) }\end{array}$ & MTX, TNFi & $\begin{array}{l}\text { SORBS3, AKAP9, } \\
\text { CYP4F12, MUSTN, } \\
\text { CX3CR1, SLC2A3, } \\
\text { C21orf58,TBC1D8 } \\
\text { sICAM1, CXCL13 } \\
\text { rs6028945 } \\
\text { rs7305646 InCRNA }\end{array}$ & $\begin{array}{l}\text { ICAM1 } \\
\text { chemokines }\end{array}$ & $\begin{array}{l}\text { The combined variables explain half of } \\
\text { the variation in } \triangle D A S 28-C R P \text { in the TNFi } \\
\text { group. Most of the prediction comes } \\
\text { from sICAM1, CXCL13, CX3CR1 and } \\
\text { SLC2A3 } \\
\text { (EULAR criteria, deltaDAS28-CRP at } 3 \\
\text { months) }\end{array}$ \\
\hline \multicolumn{6}{|c|}{ TRANSCRIPTS PROTEINS, IMMUNE CELLS } \\
\hline $\begin{array}{l}\text { Tasaki S (2018) } \\
\text { [157] }\end{array}$ & $\begin{array}{l}\text { RA (45) } \\
\text { HC (35) }\end{array}$ & MTX IFX TCZ & $\begin{array}{l}\text { whole blood } \\
\text { transcriptome, serum } \\
\text { proteome, cell counts }\end{array}$ & $\begin{array}{l}\text { Neutrophils, } \\
\text { NK } \\
\text { complement } \\
\text { (C3) }\end{array}$ & $\begin{array}{l}\text { After treatment: decreased neutrophils, } \\
\text { increased NK (gene expression and cell } \\
\text { counts), normalization of complement } \\
\text { pathway proteins }\end{array}$ \\
\hline
\end{tabular}

Abbreviations: ABA, abatacept; ADA, adalimumab; CTZ, certolizumab; ETN, etanercept; GOL, golimumab; IFX, infliximab; MTX, methotrexate; DMARD, disease-modifying antirheumatic drugs; TNFi, TNF-inhibitors; HC, healthy controls; Rs, responders; NRs, non-responders; NLR, neutrophils/lymphocyte ratio; PLR: 
platelet/lymphocyte ratio, CRP, C-reactive protein; ESR, erythrocyte sedimentation rate; DAS28, disease activity score 28; SJC, swollen joint counts, EULAR, European League Against Rheumatism.

A recent community-based effort has assessed the use of available SNP data to predict clinical outcome of TNF inhibition in RA patients, using machine learning algorithms [107]. Despite a significant estimate of heritability of the treatment response, none of the proposed models could demonstrate a significant contribution of genetic variants to response prediction, over the conventional clinical parameters [107]. A useful strategy to improve the power of the predictive models could be the combination of different types of biomarkers that better capture the non-genetic components of response to therapy.

In the context of IBD, an association study highlighted polymorphisms in the TLR2 gene that are associated with response to anti-TNF therapy. rs3804099 and rs1816702 were associated with beneficial response to treatment in CD patients, suggesting that genetically determined increased TLR2 levels were associated with beneficial response among patients with CD [50]. However, the rs11938228 and rs4696480 polymorphism at the TLR2 locus were associated with nonresponse to treatment in the UC patients [50]. Another recent study from Bank et al. showed that polymorphisms in genes involved in the regulation of the NFKB pathway (TLR2, TLR4, and NFKBIA), the TNF- $\alpha$ signaling pathway (TNFRSF1A), and other cytokine pathways (NLRP3, IL1RN, IL18, and i) were associated with primary response to anti-TNF therapy in IBD patients [49].

Another recent genetic association study in two independent large IBD cohorts successfully replicated 2 variants (rs116724455 in TNFSF4/18, rs2228416 in PLIN2), which may predict anti-TNF response in patients with IBD at genome-wide significance. The minor alleles of the two polymorphisms were associated with refractory response to anti-TNF agents [158].

\subsection{Epigenetic biomarkers}

Pharmacoepigenetic profiling may also add another layer of complexity to the relation between genetics and variations in drug responses [159]. Only a few studies in the literature have explored the epigenetic modifications of chromatin before and after anti-TNF treatment in order to identify epigenetic biomarkers that may predict treatment responses in patients affected by rheumatic diseases. To date, most of the epigenomic studies were designed to identify diagnosis biomarkers or new therapeutic targets, and only few were aimed at the identification of biomarkers predictive of anti-TNF outcome. In an attempt to identify epigenetic biomarkers that could predict response to biological drugs, including adalimumab, in psoriasis and psoriatic arthritis patients, Ovejero-Benito et al. assessed 4 histone modifications (H3 and $\mathrm{H} 4$ acetylation, H3K4 and H3K27methylation) in PBMC of 39 psoriasis patients using an ELISA technique, before and after treatment initiation. Significant changes in histone $\mathrm{H} 3$ and $\mathrm{H} 4$ acetylation and H3K4 methylation were observed between psoriasis patients and controls, however, no changes in histone 
modification were seen upon treatment, except for a decrease in H3K27 methylation in responders compared to non-responders [78]. Although this study presents many limitations, including the fact that the small number of patients were treated with different biologicals and that a mixture of immune cell populations was analyzed, it is one of very few studies that tried to identify epigenetic biomarkers to predict treatment outcome in rheumatic diseases, and it set the basis for other similar studies on larger cohorts.

Most of the epigenetic studies performed on SpA have focused on miRNA, very few on DNA methylation and even fewer on histone modifications [160]. miRNAs are regulatory non-coding RNA molecules; their expression may be modified under pathophysiological stress conditions, disease or treatment [161]. In a small exploratory study, miRNA-5196 expression levels in serum were measured using real-time PCR. The analysis of samples from 10 RA patients and 13 AS patients, before and after antiTNF- $\alpha$ therapy, revealed that changes in miRNA-5196 expression positively correlated with the decrease in disease activity scores in patients following anti-TNF- $\alpha$ therapy, suggesting that MiRNA-5196 may be an interesting biomarker to assess treatment response [79]. In silico analysis identify the Fra2 transcription factor, Matrix Metalloproteinase 15 (MMP-15) and the IL-1 receptor as possible targets of MiRNA-5196. The possible biological role of MiRNA-5196 in AS and RA, as well its utility as a clinical biomarker, need confirmative replication studies in larger cohorts.

Baseline serum or whole blood MiRNA signature associated with treatment responses have also been explored in RA patients treated with TNF-blockers. Among the putative targets of differentially expressed MiRNA were molecules of the IL6 and NF-kB pathways, chemokines, and extracellular matrix proteins $[112,113]$. Although these studies included a larger number of patients (Table 2), validation in a replication cohort is still lacking.

In a recent study, Tao et al. analyzed both gene expression and DNA methylation profiles in peripheral blood mononuclear cells, and in CD4+ T cells and monocytes isolated from RA patients before anti-TNF treatment [154]. The authors identified a large number of genes differentially expressed (DEG) between responders and non-responders at baseline, as well as differentially methylated regions (DMR), although the majority of DEG and DMR were not significant after correction for multiple testing, and the overlap of DEG with those identified previously was small [162]. Notably, both DEG and DMR were specific not only to the cell population studied, but also to the type of anti-TNF treatment (etanercept versus adalimumab). Among the DEG identified in this study were transcription factors (such as IRF1, FOXO3 and FOXO4), genes of the TNF and JAK/STAT signaling pathways, and IL18R1. The authors applied random forest-based algorithms with internal cross-validation, in order to construct predictive models for response to adalimumab or etanercept. The models were subsequently tested in a small replication cohort (9 patients) and in patients assigned to a second TNFi treatment. The prediction accuracy of the models were different for each cell population, treatment and type of data, and the model based on methylation data for response to adalimumab reached the highest accuracy of $88 \%$ [154]. Although these results require 
replication in an independent patient cohort, this study underlines the importance of exploring several approaches to identify predictive biomarkers, and of analyzing patients treated with different drugs separately.

Spiliopoulos et al. also tested different parameters to construct predictive models for response to TNFi in RA patients [155]. The authors took advantage of the new GENOSCORE platform to create genetic scores for RA associated loci, for immune cell traits and for the expression or methylation of a set of genes whose expression levels were previously associated with treatment response. The genotypic scores for each type of intermediate trait was evaluated for its ability to improve predictive models when added to clinical parameters. Genotypic scores for RA risk improved response prediction, however explained less than $1 \%$ of the variance in phenotype, suggesting only a marginal overlap of the genetics of RA disease risk and of response to TNFi. The effect was driven mainly by regional score at the CD40 locus. Among the scores for immune cell traits, expression levels of CD39 on CD4+ T cells were associated with worse response. The inclusion of scores linked to expression (eQTL) quantitative trait loci also resulted in a small improvement of the prediction performance of the model, but further addition of methylation QTLs had no effect, suggesting that eQTLs and mQTLs provide overlapping information.

Overall, integrative approaches for prediction of anti-TNF treatment outcome are still poorly explored. A proof of concept for this strategy in RA patients was provided by Folkersen et al., who constructed prediction models based on selected SNP, transcripts or protein biomarkers which had been associated with response to therapy in the literature [156]. The model combining all three different type of biomarkers explained 51\% of the variation in DAS28-CRP after TNFi therapy, and resulted in an improved AUC, compared to the model with protein biomarkers alone [156].

\subsection{Cellular profiling}

Explorative studies to identify cell-based biomarkers that can be used to predict response to TNF inhibitors have used flow cytometry-based approaches to identify qualitatively and/or quantitatively modified cell populations before and after treatment onset.

An interplay of several innate and adaptive immune cell populations may be involved in SpA pathogenesis [163], and it has been reported that TNFi impact T cell populations in SpA patients [94-97]. All these observations suggest that analyzing changes in immune cells populations in SpA patients undergoing treatment may be of interest to explore disease mechanisms and therapeutic correlations.

Ursula Schulte-Wrede et al [93] performed a deep profiling of peripheral leukocytes in 31 AS patients under adalimumab or etanercept, using an integrated, multi-parametric flow cytometric approach to analyze 50 different surface antigens. The authors then applied an automated cell clustering approach (immunoClust) to identify immunophenotypic signatures predictive of response. The baseline frequencies of CD8+ NK cell subsets turned out to carry the best predictive power for therapeutic outcome in AS patients, with pre-treatment frequency of CD8+ NK cells significantly higher in responders compared 
with non-responders, in particular for etanercept-treated patients. Although further validation of NK cell subsets for clinical prediction of TNF inhibitor outcome is necessary, this study suggests that cellular response signatures can be identified in peripheral blood using extensive immunophenotyping approaches [93].

Increased effector responses after TNFi treatment has also been observed in RA patients (Table 2). Patients treated with golimumab showed an increase in the CD8+ T cell effector population, accompanied by increased responses to viral antigens, suggesting that TNF blockade, while broadly suppressing inflammation, does not induce generalized immunosuppression, but rather may "normalize" immune responses [34]. In agreement with this concept, anti-TNF treatment did not affect the expression of TNF-inducible genes in the site of acute inflammatory challenge (tuberculin injection), while decreasing the inducibility of these genes in blood samples [116].

Many studies have suggested a role for effector T cell populations such as Th1 and Th17 in SpA pathogenesis and disease activity [164]. In a targeted cellular profiling approach, some studies focused on specific effector cell subsets, such as Th1, Th17, and Treg cell populations. The evaluation of these cell subsets before and after anti-TNF treatment is so far rather inconclusive, perhaps due to the difficulties of reliably quantifying small cell populations by immunophenotyping large cohorts of patients. Xueyi et al [95] analysed Th17 and Treg populations in 222 AS patients at baseline and after anti-TNF treatment. At baseline, significantly higher Th17 frequencies and lower Treg frequencies were observed relative to healthy controls, independently of future response to treatment. Baseline frequencies of Th17 cells, and baseline levels of Th17-related cytokines were positively correlated with the BASDAI disease score. After anti-TNF therapy Th17 cell frequencies declined significantly only in responders, while increasing in nonresponders. In the same study, the authors found that also serum levels of IL-6, IL-17 and IL-23 significantly decreased only in responders [95]. On the contrary, an increase in IL-17+ and in IL-22+ cells after adalimumab treatment was reported by Andersen et al. in $30 \mathrm{SpA}$ patients [96].

An increase of Th17 frequency in patients' blood after TNF inhibition was also observed in a study on 25 RA patients. This study also reported that higher Th17 frequencies at baseline correlated with worse response to anti-TNF therapy, as defined by ultrasonographic measure of synovial thickening [145].

Alterations in blood cell counts may occur in inflammatory disorders, and ratios of circulating blood cell components can be used to assess inflammatory activity. Neutrophil-lymphocyte ratio (NLR), monocyte-lymphocyte ratio (MLR) and platelet-lymphocyte ratio (PLR) have been found to correlate with the erythrocyte sedimentation rate (ESR) and C-reactive protein (CRP) levels, suggesting their use as markers of systemic inflammation for the prognosis of chronic inflammatory diseases [91,165]. Enginar and Kacar [91] retrospectively evaluated 68 RA patients and 203 AS patients on anti-TNF medication for at least 6 months. NLR and PLR correlated with disease activity in both RA and SpA patients. Other studies performed on RA and AS patients have also found similar results $[166,167]$. 
Despite growing evidence of the association of NLR and PLR with disease activity, the association of baseline NLR and PLR with response to treatment is less clear. In UC patients with moderate to severe disease, high baseline neutrophil to lymphocyte ratio (NLR), reflecting a high neutrophil count, is an indicator of loss of response to infliximab in patients with moderate-to-severe active UC [168].

Similarly, Han-Na Lee et al. found that, in 82 RA patients, high baseline NLR and PLR were independently associated with a higher risk of non-response to anti-TNF treatment at 12 weeks, but not at 24 weeks [147].

On the other hand, Farutin et al., in a combined whole blood transcriptome and plasma proteome analysis of RA patients, identified the downregulation of myeloid cells and platelet genes as a molecular signature of anti-TNF treatment. This signature was present in both responders and non-responders. However, at baseline, the top differentially expressed genes between responders and non-responders included markers of myeloid cells, which were higher in good responders. The association between higher NLR and good response was also confirmed by the analysis of the NLR in a cohort of 1962 RA patients [153]. The discrepancies observed in the relationship of the NLR with therapeutic responses may be due not only to the limited number of patients in some studies, but also to heterogeneity in the timing of the observation, and in the measures of clinical outcome. As cellular ratios are simple to measure, inexpensive, and easily accessible parameters, further exploring their significance in response prediction may still be a reasonable strategy.

A particularly informative approach may be the analysis of cell populations in the inflamed tissue, when obtaining tissue biopsies is possible. The combined immunohistochemical and gene expression analysis of synovial tissue from early RA patients showed that detection of a lympho-myeloid pathotype before treatment is significantly associated with disease progression requiring biologics at 12 months from the diagnosis [151]. Several synovial gene expression modules, including type I IFN and antiviral modules, $B$ cells and dendritic cell modules, correlated with response to treatment [26].

In ileal Crohn's disease patients, a recent single cell analysis of the inflamed ileal tissue indicated that a subgroup of patients presented a distinct cellular pattern, called the GIMATS module, which included IgG+ plasma cells, inflammatory macrophages and activated dendritic cells, activated T cells, and stroma cells (activated fibroblasts, and endothelial cells). The enrichment of this cellular module before anti-TNF therapy was predictive of resistance to anti-TNF therapy [169].

\subsection{Protein biomarkers}

Studies relative to protein biomarkers for TNF inhibitors treatment in SpA have been largely focused on candidate proteins selected for their known role in inflammation and/or bone metabolism, such as acute phase proteins or cytokines. Many studies have shown that anti TNF treatments are effective in modulating acute inflammation proteins [83,170-172]. However, if the baseline serum levels of these proteins can be used to prospectively predict response to treatment needs to be confirmed. In addition, 
these markers may lack specificity and be elevated in other inflammatory processes unrelated to $\mathrm{SpA}$. To date, CRP is the most important marker of inflammation in rheumatology and is the only biomarker currently used in clinical practice to select SpA patients for treatment and to predict TNFi therapy outcome [173]. Using a large dataset of 867 AS patients treated with Etanercept, Baraliakos et al. found that categorization of CRP levels prior to treatment initiation is a highly significant predictor of treatment outcome at 12 weeks, with higher baseline CRP categories predictive of a greater proportion of responders, and decreases from very high to normal CRP levels after treatment start, predictive of the future course of TNFi treatment [87].

Many studies have shown that anti TNF treatments are effective in modulating acute inflammation proteins $[83,170-172]$ and several studies attempted to correlate baseline serum levels of biomarkers in TNF naïve patients with treatment outcome [170]. However, at date there is no robust replicated evidence that permits to extrapolate the results to all SpA patients, in particular because baseline inflammatory protein levels fluctuate among patients and depend on disease activity status. An alternative approach is the use of short-term changes in serum biomarkers levels upon anti-TNF treatment to predict long term response to treatment. A common conclusion for both approaches is that combining markers yields a stronger predictive power than the use of an individual marker [65].

Wagner et al. tested serum samples collected at baseline, 4 and 14 weeks from 100 active AS patients randomized to receive golimumab. Selected inflammatory, bone and cartilage markers were analyzed, and profiling of 92 different proteins was performed [81]. The levels of many of these markers was affected by TNF inhibition, including a number of acute phase reactants, bone metabolism factors, coagulation factors (eg. C3), inflammatory chemokines (eg. RANTES and MIP1 $\beta$ ), matrix metalloproteinase pathways (eg. MMP3). A robust logistic regression analysis associated baseline biomarkers levels of insulin, leptin, apolipoprotein C3, IL-6, osteocalcin, N-terminal propeptide of type 1 collagen (P1NP) with ASAS 20 response at week 14 . The combination of baseline levels of insulin and P1NP was a stronger predictor of response than CRP levels [81].

Leptin, IL-6 and MMP3 are also included in the 12-proteins Multi-Biomarker Disease Activity score, or MBDA. The MBDA score was originally aimed to assess disease activity, and was developed through a multi-step approach of candidate biomarker prioritization and algorithm development [121]. Of the 130 putative protein biomarkers tested, 12 were retained in the final multi-biomarker statistical model. Eleven of these (tumor necrosis factor receptor I (TNF-RI), IL-6, vascular cell adhesion molecule 1 (VCAM-1), epidermal growth factor (EGF), VEGF-A, cartilage glycoprotein 39 (YKL40), matrix metalloproteinase 1 (MMP1), MMP3, serum amyloid A (SAA), leptin, and resistin) were modelled to predict disease activity scores, and then combined with CRP to produce the final MBDA score. These proteins are involved in a variety of innate and adaptive immune pathways, in systemic inflammation and bone/cartilage metabolism [121]. The MBDA score was significantly correlated with Disease Activity Score 28-CRP (DAS28- 
(RP), as well as with additional measures of joint inflammation and structural progression by ultrasonography and radiography [122].

The potential of the MBDA score in predicting disease progression has also been extensively analyzed (Table 2), with low MBDA score, in particular, showing good negative predictive power for structural progression [133]. MBDA scores at baseline have been associated with patients' response to anti-TNF therapy (Table 2). On the other hand, a randomized prospective study did not find a correlation between MBDA score and different clinical scores in patients treated with adalimumab or abatacept [125], and the contribution of the MBDA score to personalized patient management is still under discussion [174-176]. The score may perhaps find its most informative application in the detection of subclinical inflammation in patients with low disease activity by conventional parameters, such as patients in DAS28-CRP remission [177], or low CRP [124], and may therefore play a complementary role to these measures.

Calprotectin is another inflammation marker particularly studied in CID such as SpA, RA, and IBD. Calprotectin may be a promising candidate biomarker to monitor disease activity, bone damage progression and predict response to anti-TNF therapy [178]. This protein is a heterodimeric complex of the S100A8 and S100A9 proteins (also called myeloid-related protein MRP8 and MRP14), and is a damage associated molecular pattern (DAMP) that triggers the innate immunity receptor TLR4, and the Receptor for Advanced Glycation Endproducts (RAGE) [179]. Calprotectin is produced by monocytes and neutrophils locally, suggesting it may be a good marker of tissue inflammation. Both serum and faecal calprotectin levels are increased in SpA patients compared to healthy controls and are associated with CRP, ESR, and disease scores in SpA [85]. Serum calprotectin levels decreased with anti TNF treatment and early changes in calprotectin levels could predict patients achieving ASAS40 with an AUC of 0.691 [85]. In a similar study on faecal calprotectin, $\varnothing$ stgård et al. found that high faecal calprotectin levels accurately identified SpA patients with intestinal inflammation. This group of patients was more likely to respond better to Adalimumab treatment, as evaluated by changes in the ASDAS. Faecal calprotectin also associated with stronger inflammation in the sacroiliac joint, as shown by MRI [84]. Turina et al identified Calprotectin as the most promising biomarker of treatment response in SpA and PsA patients, compared to high-sensitive CRP (hsCRP), MMP3 and IL-6. With the highest sensitivity to change upon clinical effective treatment, calprotectin outperformed hsCRP, suggesting it may be a useful maker of disease in CRP-negative patients [83]. Whether baseline concentrations of calprotectin in serum, synovial fluid or faeces can predict treatment responses needs to be confirmed by replication studies on larger cohorts and using standardized methodologies.

Also in RA, serum levels of calprotectin correlate well with disease activity, and are potentially a predictive marker for response (Table 2). Higher baseline calprotectin levels were found to correlate with treatment response [135,136], with relapse after remission [139] and flares after tapering or discontinuation of TNF blockers [142]. However, inconsistencies for this marker have also been reported, 
since some studies failed to find a correlation with clinical response [138], or obtained different results in different cohorts [140].

Matrix Metalloproteinases (MMPs), and MMP3 in particular, are reported biomarkers of synovial inflammation and cartilage turnover in inflammatory joint diseases, such as SpA and RA [180]. MMP3 baseline serum levels are elevated in SpA patients compared to healthy controls and correlate with disease activity [181-183]. In the Turina et al study [83], MMP3 showed a statistically significant decrease after two weeks of anti-TNF treatment, however, the effects of treatment were modest and less consistent across different subtypes of SpA. The impact of TNF inhibition on MMP3 levels is so far inconclusive with opposing results from different studies [184], raising the question of whether MMP3 can be used as a predictive biomarker for anti-TNF response. Some studies found a good predictive value for either baseline serum levels and/or reduction over time of MMP3 in SpA and PsA patients under anti-TNF [185,186], Arends et al. [80], however, showed that changes in MMP-3 levels after treatment correlated with disease activity, but baseline MMP3 levels had no predictive value for treatment response.

Tissue biomarkers are OSM (encoding the cytokine oncostatin-M) and its receptor OSMR, which were overexpressed in the intestinal mucosa of patients with active IBD ( 5 different cohorts, 227 patients) [187]. Neither OSM nor OSMR expression was correlated with standard clinical parameters, including CRP. However, OSM and OSMR expression was increased in patients with IBD who required surgery. High baseline OSM expression in the intestinal mucosa was reproducibly associated with resistance to anti-TNF therapy in IBD [187]. Two additional studies found that high plasma OSM was associated with nonresponse to anti-TNF treatment in CD patients $[188,189]$. Oncostatin $M$ is a cytokine produced by $T$ cells and innate immune cells, including monocytes and neutrophils. The OSM receptor is expressed on nonhematopoietic cells, such epithelial cells, fibroblasts and endothelial cells, and the OSM/OSMR axis may therefore be crucial for the cross-talk between stroma and immune cells [190].

\section{Conclusions}

The past years have been marked by a major increase of our understanding of the cellular and molecular mechanisms of TNF-blocker action in patients affected by chronic inflammatory diseases. However, despite substantial efforts, a validated biomarker predicting therapeutic responses of patients to TNFi has not yet been identified.

There are several reasons that may explain our limited success to identify validated biomarkers that can inform treatment choices. Many studies reported in the literature have been underpowered and patients have not been appropriately stratified. An important issue that is more difficult to tackle is that currently used clinical outcome measures, in particular for SpA, extensively rely on self-reporting. It will be of critical importance to move from self-reporting to objective and quantifiable outcome measures. Furthermore, it is now well appreciated that the biology of treatment failures and development of 
resistance to treatment is highly complex, as it is influenced by multiple patient-intrinsic, drug-specific and environmental parameters. To account for this complexity, it may be necessary to develop multidimensional biomarkers. However, the general use of biomarkers in the clinics requires that they rely on robust and inexpensive, non-invasive procedures.

Several biomarker candidates to predict treatment responses have emerged recently, in particular OSM and IL7R, whose expression is negatively correlated with response to anti-TNF therapy in IBD. Further studies are needed to validate these candidates, and to test their potential relevance for other diseases.

\section{Expert Opinion}

The introduction of biologic therapies, such as TNF-blockers, has been a major breakthrough in the treatment of CID. However, these therapies are effective only in a sub-population of patients, and it is currently not possible to predict which patients will not respond to the treatment. In clinical practice, patients non-responsive to a TNF-blocker are treated with a different TNF inhibitor until an effective therapeutic agent is identified. This procedure is expensive and may take a long time, during which the patient is not appropriately treated and is exposed to side effects without clinical benefit. The negative impact of unsuccessful therapy on patients' quality of life, adds to the negative impact on society, due to the associated high costs of treatment.

A reason for the lack of a "personalized" approach to treatment is the insufficient understanding of what determines the individual predisposition to disease and the mechanisms associated with the response to a specific therapy. In the past years there have been multiple efforts to identify biomarkers that predict response to biological therapies in SpA, RA, IBD and other CID. The results have been, however, rather limited. Some of these biomarkers appear to be highly promising (for example Oncostatin $\mathrm{M}$, in the case of (BD), however, none has been validated yet as a tool to inform treatment choices. The search for biomarkers that correlate with disease activity has been more successful: the Endoscopic Healing Index (EHI) Score has been developed commercially as a tool to monitor disease activity in Crohn's disease patients, and the MBDA blood test is commercialized as a biomarker for disease activity in RA, although their use is still considered investigational. These biomarkers have also been proposed to predict treatment outcome, but this application remains to be validated.

Several hurdles to the prediction of treatment responses have emerged, such as the limited size and the heterogeneity of cohorts, in terms of patients' characteristics and treatment (for example, patients treated with different anti-TNF compounds are often analyzed together). There is also a lack of adequate replication studies in different patient populations. In addition, a key issue is the suitability of the current clinical outcome measures, in particular for SpA. The BASDAI score is exclusively based on subjective parameters of self-reporting, and the ASDAS, is a composite of self-reporting and CRP levels. In the case of RA, the DAS28 may represent a more robust outcome measure, since it combines the subjective 
measure of tender joint counts (TJC) with the objective observation of swollen joints (SJC). However, even the DAS28 may not constitute a reliable end-point measure [45]. In addition, these scores are usually determined at single time points: integration of longitudinal monitoring would be needed to smoothen score fluctuations.

Genetic biomarkers are attractive, because they are robust, do not vary and are easily measured. However, none of the proposed candidates have been validated to date: the identification may be hindered by the small effect size of common SNPs, which would require studies on very large cohorts to determine an extended panel of SNPs that, combined, have an impact on treatment outcome. Additional complications for the use of SNPs as response biomarkers are the effects of population ancestry, and the fact that they do not address the non-genetic contribution to disease and response to therapy.

Blood biomarkers are ideal for clinical applications, for the ease of sampling. This is especially true for pathologies (such as axSpA) where routine access to diseased tissues is difficult. To date, no robust blood response biomarker has been identified in SpA and in other CID, possibly because of the fluctuation of biomarker levels with time, and the fact that they may not quantitatively reflect pathological processes in tissue.

Immune biomarkers are also strongly impacted by many variables such as age and sex, or environmental factors (for example smoking or CMV infection), introducing another layer of variability that has to be taken into account in the study design, by providing adequate patient stratification.

For the years to come, a key issue will be the constitution of large and highly annotated longitudinal cohorts. These resources are now widely available in the cancer field (examples are The Cancer Genome Atlas, TCGA, and the International Cancer Genome Consortium, ICGC). Standardization of procedures for the collection and management of demographic and clinical data will facilitate comparison of biomarker studies performed in different clinical centers. In addition, the importance of the application of standardized and robust methodology for sample analysis cannot be overstated.

Finally, the combination of different biomarkers that capture the biological complexity of the disease and of the effect of treatment may be necessary to successfully stratify patients to the most appropriate treatment. In this respect, we believe that the combination of multi-dimensional biomarkers and the development of mathematical models (such as deep learning tools) to capture all the biological/environmental influences on treatment outcomes will be an important driver of progress in this area. Finally, it will be important to move towards "objective" outcome measures (including imaging technology) and molecular biomarkers to develop objective and reliable criteria to help clinical decision making. 


\section{Figure legends}

Figure 1. Design of gene modules for Quantitative Set Analysis for Gene Expression (QuSAGE).

The design the 45 gene modules was based on the deconvolution of cell signaling pathways. The NF-kB signaling pathway was decomposed to create modules dedicated to the genes coding for transmembrane proteins forming the receptors and the genes encoding their ligands. Other modules group intermediate intracellular kinases (NF-kB regulators), which, once activated, phosphorylate IkB $\alpha$ (inhibitor of NF-kB $\alpha$ subunit), and NF-kB inhibitors and transcription factors.

Figure 2. Analysis of signaling pathways reveals multiple mechanisms of TNF-blocker action in SpA.

Shown are the effects of anti-TNF therapy on the "pathway activity" of 45 gene modules generated from 594 immune-related genes. Peripheral blood samples were obtained from 17 axSpA patients before (D0) and 7 days (D7) after the first injection of a TNF-blocker. Whole-blood cultures were stimulated with Candida albicans and RNA was extracted after a 22-hour culture. Gene expression was measured using Immunology_v2 nCounter panel (Nanostring). For each gene module, the mean activity fold-change and 95\% confidence interval are plotted and color-coded according to their FDR-corrected P-values (means compared to fold-change zero). Confidence intervals overlapping the horizontal dotted line indicate statistically significant increased or decreased module activity at D7 as compared to D0.

Figure 3. Detailed gene activity for 3 representative modules.

Shown is the "gene activity" for individual genes from 3 modules shown in Fig. 2. A, Cytotoxic molecules; B, M2 macrophages; and C, Pro-inflammatory molecules. Whole-blood cultures were stimulated with Candida albicans as in Fig. 2. The horizontal dashed blue line and the grey band indicate the mean differential expression of all genes in the module at D7 versus D0, and the $95 \%$ confidence interval.

Figure 4. A gene expression signature associated with therapeutic responses to anti-TNF therapy in axSpA.

Shown is a heat map representation of genes differentially expressed between axSpA patients with a major response to anti-TNF therapy (delta ASDAS $\geq 2$, green squares) and non-responders (delta ASDAS $<1.1$, red squares) in whole-blood cultures stimulated with LPS or SEB before initiation of therapy. The heatmap shows the levels of differentially secreted genes (red indicates higher and green lower levels of protein secretion, LIMMA analysis, adjusted p-value < 0.05). Gene-stimulus combinations were ranked by decreasing fold-change. Expression levels and fold-change values of the 58 gene-stimulus combinations (corresponding to 55 genes) that are the most differentially expressed between responders and nonresponders are reported in [28]. 


\section{Funding}

I.M. is supported by the Pasteur - Paris University (PPU) International PhD Program. Work in the authors' laboratory is supported by grants from Institut Pasteur, FOREUM Foundation for Research in Rheumatology, MSD Avenir (Project iCARE-SpA), and a Sanofi Innovation Award Europe.

\section{Declaration of interest}

The authors declare no competing financial interest.

\section{ORCID}

Ikram Mezghiche: https://orcid.org/0000-0001-7730-3251

Hanane Yahia-Cherbal: https://orcid.org/0000-0002-6288-5209

Lars Rogge: http://orcid.org/0000-0003-1262-9204

Elisabetta Bianchi: https://orcid.org/0000-0001-6612-8881

\section{References}

[1] El-Gabalawy H, Guenther LC, Bernstein CN. Epidemiology of immune-mediated inflammatory diseases: incidence, prevalence, natural history, and comorbidities. J Rheumatol Suppl. 2-1085 (2010).

[2] Jacobs P, Bissonnette R, Guenther LC. Socioeconomic burden of immune-mediated inflammatory diseases--focusing on work productivity and disability. J Rheumatol Suppl. 55-6188 (2011).

[3] Braun J, Listing J, Sieper J. Reply. Arthritis Rheum. 4049-405052 (2005).

[4] Taurog JD, Chhabra A, Colbert RA. Ankylosing Spondylitis and Axial Spondyloarthritis. N Engl J Med. 2563-2574374 (2016).

[5] Sieper J, Braun J, Dougados M, et al. Axial spondyloarthritis. Nat Rev Primer. 150131 (2015).

[6] Ermann J, Rao DA, Teslovich NC, et al. Immune cell profiling to guide therapeutic decisions in rheumatic diseases. Nat Rev Rheumatol. 541-55111 (2015).

[7] Reveille JD. Biomarkers for diagnosis, monitoring of progression, and treatment responses in ankylosing spondylitis and axial spondyloarthritis. Clin Rheumatol. 1009101834 (2015).

[8] Heiberg MS, Koldingsnes W, Mikkelsen K, et al. The comparative one-year performance of anti-tumor necrosis factor alpha drugs in patients with rheumatoid arthritis, psoriatic arthritis, and ankylosing spondylitis: results from a longitudinal, observational, multicenter study. Arthritis Rheum. 234-24059 (2008). 
[9] Baeten D, Sieper J, Braun J, et al. Secukinumab, an Interleukin-17A Inhibitor, in Ankylosing Spondylitis. N Engl J Med. 2534-2548373 (2015).

[10] Elliott MJ, Maini RN, Feldmann M, et al. Treatment of rheumatoid arthritis with chimeric monoclonal antibodies to tumor necrosis factor alpha. Arthritis Rheum. 1681-169036 (1993).

[11] Feldmann M, Maini RN. Anti-TNF alpha therapy of rheumatoid arthritis: what have we learned? Annu Rev Immunol. 163-19619 (2001).

[12] Present DH, Rutgeerts P, Targan S, et al. Infliximab for the treatment of fistulas in patients with Crohn's disease. N Engl J Med. 1999/05/06. 1398-1405340 (1999).

[13] van Dullemen HM, van Deventer SJ, Hommes DW, et al. Treatment of Crohn's disease with anti-tumor necrosis factor chimeric monoclonal antibody (cA2). Gastroenterology. 1995/07/01. 129-135109 (1995).

[14] Brandt J, Haibel H, Cornely D, et al. Successful treatment of active ankylosing spondylitis with the anti-tumor necrosis factor alpha monoclonal antibody infliximab. Arthritis Rheum. 1346-135243 (2000).

[15] Menegatti S, Bianchi E, Rogge L. Anti-TNF Therapy in Spondyloarthritis and Related Diseases, Impact on the Immune System and Prediction of Treatment Responses. Front Immunol. 38210 (2019).

[16] Chaudhari U, Romano P, Mulcahy LD, et al. Efficacy and safety of infliximab monotherapy for plaque-type psoriasis: a randomised trial. Lancet. 2001/06/19. 18421847357 (2001).

[17] Mease PJ, Goffe BS, Metz J, et al. Etanercept in the treatment of psoriatic arthritis and psoriasis: a randomised trial. Lancet. 2000/09/06. 385-390356 (2000).

[18] Charles P, Elliott MJ, Davis D, et al. Regulation of cytokines, cytokine inhibitors, and acute-phase proteins following anti-TNF-alpha therapy in rheumatoid arthritis. $\mathrm{J}$ Immunol. 1521-1528163 (1999).

[19] Taylor PC, Peters AM, Paleolog E, et al. Reduction of chemokine levels and leukocyte traffic to joints by tumor necrosis factor alpha blockade in patients with rheumatoid arthritis. Arthritis Rheum. 2000/01/22. 38-4743 (2000).

[20] Paleolog EM, Young S, Stark AC, et al. Modulation of angiogenic vascular endothelial growth factor by tumor necrosis factor alpha and interleukin-1 in rheumatoid arthritis. Arthritis Rheum. 1998/07/15. 1258-126541 (1998).

[21] Tracey D, Klareskog L, Sasso EH, et al. Tumor necrosis factor antagonist mechanisms of action: a comprehensive review. Pharmacol Ther. 244-279117 (2008). 
[22] Wang XB, Ellis JJ, Pennisi DJ, et al. Transcriptome analysis of ankylosing spondylitis patients before and after TNF-alpha inhibitor therapy reveals the pathways affected. Genes Immun. 184-19018 (2017).

[23] Xu L, Sun Q, Jiang S, et al. Changes in gene expression profiles of the hip joint ligament of patients with ankylosing spondylitis revealed by DNA chip. Clin Rheumatol. 1479149131 (2012).

[24] Goedecke V, Crane AM, Jaakkola E, et al. Interleukin 10 polymorphisms in ankylosing spondylitis. Genes Immun. 74-764 (2003).

[25] Tsukazaki H, Kaito T. The Role of the IL-23/IL-17 Pathway in the Pathogenesis of Spondyloarthritis. Int J Mol Sci. 640121 (2020).

[26] Lewis MJ, Barnes MR, Blighe K, et al. Molecular Portraits of Early Rheumatoid Arthritis Identify Clinical and Treatment Response Phenotypes. Cell Rep. 2455-2470.e528 (2019).

[27] Duffy D, Rouilly V, Libri V, et al. Functional Analysis via Standardized Whole-Blood Stimulation Systems Defines the Boundaries of a Healthy Immune Response to Complex Stimuli. Immunity. 436-45040 (2014).

[28] Menegatti S, Guillemot V, Latis E, et al. Immune response profiling of patients with spondyloarthritis reveals signalling networks mediating TNF-blocker function in vivo. Ann Rheum Dis. (2020).

** The study uses gene pathway analysis to explore the impact of anti-TNF therapy on stimulated immune responses in SpA. Differential expression of genes in responders versus non-responders before treatment suggests this approach may identify signaling pathways associated with response to therapy.

[29] Nielsen T, Wallden B, Schaper C, et al. Analytical validation of the PAM50-based Prosigna Breast Cancer Prognostic Gene Signature Assay and nCounter Analysis System using formalin-fixed paraffin-embedded breast tumor specimens. BMC Cancer. 17714 (2014).

[30] Yaari G, Bolen CR, Thakar J, et al. Quantitative set analysis for gene expression: a method to quantify gene set differential expression including gene-gene correlations. Nucleic Acids Res. e17041 (2013).

[31] Banchereau R, Hong S, Cantarel B, et al. Personalized Immunomonitoring Uncovers Molecular Networks that Stratify Lupus Patients. Cell. 2016/06/04. 1548-1550165 (2016).

[32] Latis E, Michonneau D, Leloup C, et al. Cellular and molecular profiling of T-cell subsets at the onset of human acute GVHD. Blood Adv. 2020/08/21. 3927-39424 (2020).

[33] Liberzon A, Birger C, Thorvaldsdottir H, et al. The Molecular Signatures Database (MSigDB) hallmark gene set collection. Cell Syst. 417-4251 (2015). 
[34] Khanniche A, Zhou L, Jiang B, et al. Restored and Enhanced Memory T Cell Immunity in Rheumatoid Arthritis After TNFa Blocker Treatment. Front Immunol. 88710 (2019).

[35] Schett G, Neurath MF. Resolution of chronic inflammatory disease: universal and tissuespecific concepts. Nat Commun. 32619 (2018).

[36] Udalova IA, Mantovani A, Feldmann M. Macrophage heterogeneity in the context of rheumatoid arthritis. Nat Rev Rheumatol. 472-48512 (2016).

[37] Bongartz T, Sutton AJ, Sweeting MJ, et al. Anti-TNF antibody therapy in rheumatoid arthritis and the risk of serious infections and malignancies: systematic review and metaanalysis of rare harmful effects in randomized controlled trials. JAMA. 2006/05/18. 2275-2285295 (2006).

[38] Keane J, Gershon S, Wise RP, et al. Tuberculosis associated with infliximab, a tumor necrosis factor alpha-neutralizing agent. N Engl J Med. 1098-1104345 (2001).

[39] Tubach F, Salmon D, Ravaud P, et al. Risk of tuberculosis is higher with anti-tumor necrosis factor monoclonal antibody therapy than with soluble tumor necrosis factor receptor therapy: The three-year prospective French Research Axed on Tolerance of Biotherapies registry. Arthritis Rheum. 2009/07/01. 1884-189460 (2009).

[40] Marino S, Cilfone NA, Mattila JT, et al. Macrophage polarization drives granuloma outcome during Mycobacterium tuberculosis infection. Infect Immun. 2014/11/05. 324 $33883(2015)$.

[41] Sieper J, Braun J, Kay J, et al. Sarilumab for the treatment of ankylosing spondylitis: results of a Phase II, randomised, double-blind, placebo-controlled study (ALIGN). Ann Rheum Dis. 1051-105774 (2015).

[42] Schett G, Lories RJ, D'Agostino MA, et al. Enthesitis: from pathophysiology to treatment. Nat Rev Rheumatol. 2017/11/22. 731-74113 (2017).

[43] Boniface K, Bak-Jensen KS, Li Y, et al. Prostaglandin E2 regulates Th17 cell differentiation and function through cyclic AMP and EP2/EP4 receptor signaling. J Exp Med. 2009/03/11. 535-548206 (2009).

[44] Atreya R, Neurath MF, Siegmund B. Personalizing Treatment in IBD: Hype or Reality in 2020? Can We Predict Response to Anti-TNF? Front Med Lausanne. 2020/09/29. 5177 (2020).

[45] Sutcliffe M, Radley G, Barton A. Personalized medicine in rheumatic diseases: how close are we to being able to use genetic biomarkers to predict response to TNF inhibitors? Expert Rev Clin Immunol. 389-39616 (2020).

[46] Machado P, Landewe R, Lie E, et al. Ankylosing Spondylitis Disease Activity Score (ASDAS): defining cut-off values for disease activity states and improvement scores. Ann Rheum Dis. 47-5370 (2011). 
[47] Machado P, Navarro-Compan V, Landewe R, et al. Calculating the ankylosing spondylitis disease activity score if the conventional c-reactive protein level is below the limit of detection or if high-sensitivity c-reactive protein is used: an analysis in the DESIR cohort. Arthritis Rheumatol. 408-41367 (2015).

[48] Swanson KV, Deng M, Ting JP-Y. The NLRP3 inflammasome: molecular activation and regulation to therapeutics. Nat Rev Immunol. 477-48919 (2019).

[49] Bank S, Julsgaard M, Abed OK, et al. Polymorphisms in the NFkB, TNF-alpha, IL-1beta, and IL-18 pathways are associated with response to anti-TNF therapy in Danish patients with inflammatory bowel disease. Aliment Pharmacol Ther. 890-90349 (2019).

[50] Bank S, Andersen PS, Burisch J, et al. Associations between functional polymorphisms in the NF $\kappa B$ signaling pathway and response to anti-TNF treatment in Danish patients with inflammatory bowel disease. Pharmacogenomics J. 526-53414 (2014).

[51] Dahlén R, Magnusson MK, Bajor A, et al. Global mucosal and serum cytokine profile in patients with ulcerative colitis undergoing anti-TNF therapy. Scand J Gastroenterol. $1118-112650$ (2015).

[52] Rismo R, Olsen T, Cui G, et al. Mucosal cytokine gene expression profiles as biomarkers of response to infliximab in ulcerative colitis. Scand J Gastroenterol. 538-54747 (2012).

[53] Belarif L, Danger R, Kermarrec L, et al. IL-7 receptor influences anti-TNF responsiveness and $\mathrm{T}$ cell gut homing in inflammatory bowel disease. J Clin Invest. 1910-1925129 (2019).

** Overexpression of IL-7R signaling pathway is associated with failure of anti-TNF treatment for $C D$ and $U C$ and this study point to IL-7R as a relevant therapeutic target and potential biomarker in clinical IBD detection and treatment.

[54] D'Haens G, Kelly O, Battat R, et al. Development and Validation of a Test to Monitor Endoscopic Activity in Patients With Crohn's Disease Based on Serum Levels of Proteins. Gastroenterology. 515-526.e10158 (2020).

[55] Xie X, Li F, Li S, et al. Application of omics in predicting anti-TNF efficacy in rheumatoid arthritis. Clin Rheumatol. 13-2337 (2018).

[56] Aterido A, Cañete JD, Tornero J, et al. A Combined Transcriptomic and Genomic Analysis Identifies a Gene Signature Associated With the Response to Anti-TNF Therapy in Rheumatoid Arthritis. Front Immunol. 145910 (2019).

[57] Barberà-Cremades M, Baroja-Mazo A, Pelegrín P. Purinergic signaling during macrophage differentiation results in M2 alternative activated macrophages. J Leukoc Biol. 289-29999 (2016). 
[58] Bours MJL, Swennen ELR, Di Virgilio F, et al. Adenosine 5'-triphosphate and adenosine as endogenous signaling molecules in immunity and inflammation. Pharmacol Ther. 358404112 (2006).

[59] Barton A, Pitzalis C. Stratified medicine in rheumatoid arthritis-the MATURA programme. Rheumatol Oxf Engl. 1247-125056 (2017).

[60] Cherlin S, Lewis MJ, Plant D, et al. Investigation of genetically regulated gene expression and response to treatment in rheumatoid arthritis highlights an association between IL18RAP expression and treatment response. Ann Rheum Dis. 1446-145279 (2020).

[61] Volin MV, Koch AE. Interleukin-18: a mediator of inflammation and angiogenesis in rheumatoid arthritis. J Interferon Cytokine Res Off J Int Soc Interferon Cytokine Res. $745-75131$ (2011).

[62] Arijs I, Quintens R, Van Lommel L, et al. Predictive value of epithelial gene expression profiles for response to infliximab in Crohn's disease. Inflamm Bowel Dis. 2090-209816 (2010).

[63] Medrano LM, Taxonera C, González-Artacho C, et al. Response to Infliximab in Crohn's Disease: Genetic Analysis Supporting Expression Profile. Mediators Inflamm. 3182072015 (2015).

[64] Arijs I, Li K, Toedter G, et al. Mucosal gene signatures to predict response to infliximab in patients with ulcerative colitis. Gut. 1612-161958 (2009).

[65] Brown MA, Li Z, Cao K-AL. Biomarker development for axial spondyloarthritis. Nat Rev Rheumatol. 448-46316 (2020).

[66] Gibson G. On the utilization of polygenic risk scores for therapeutic targeting. Barsh GS, editor. PLOS Genet. e100806015 (2019).

[67] Janiaud P, Serghiou S, Ioannidis JPA. New clinical trial designs in the era of precision medicine: An overview of definitions, strengths, weaknesses, and current use in oncology. Cancer Treat Rev. 20-3073 (2019).

[68] Jurgensmeier JM, Eder JP, Herbst RS. New Strategies in Personalized Medicine for Solid Tumors: Molecular Markers and Clinical Trial Designs. Clin Cancer Res. 4425-443520 (2014).

[69] Wray NR, Yang J, Hayes BJ, et al. Pitfalls of predicting complex traits from SNPs. Nat Rev Genet. 507-51514 (2013).

[70] Frazer KA, Murray SS, Schork NJ, et al. Human genetic variation and its contribution to complex traits. Nat Rev Genet. 241-25110 (2009). 
[71] Seitz M, Wirthmuller U, Moller B, et al. The -308 tumour necrosis factor- gene polymorphism predicts therapeutic response to TNF -blockers in rheumatoid arthritis and spondyloarthritis patients. Rheumatology. 93-9646 (2007).

[72] Liu J, Dong Z, Zhu Q, et al. TNF- $\alpha$ Promoter Polymorphisms Predict the Response to Etanercept More Powerfully than that to Infliximab/Adalimumab in Spondyloarthritis. Sci Rep. 322026 (2016).

[73] Fabris M, Quartuccio L, Fabro C, et al. The -308 TNF $\alpha$ and the -174 IL-6 promoter polymorphisms associate with effective anti-TNF $\alpha$ treatment in seronegative spondyloarthritis. Pharmacogenomics J. 238-24216 (2016).

[74] Aita A, Basso D, Ramonda R, et al. Genetics in TNF-TNFR pathway: A complex network causing spondyloarthritis and conditioning response to anti-TNF $\alpha$ therapy. Chang Y-J, editor. PLOS ONE. e019469313 (2018).

[75] Murdaca G, Gulli R, Spanò F, et al. TNF- $\alpha$ Gene Polymorphisms: Association with Disease Susceptibility and Response to Anti-TNF- $\alpha$ Treatment in Psoriatic Arthritis. J Invest Dermatol. 2503-2509134 (2014).

[76] Liu J, Zhu Q, Han J, et al. IgG Galactosylation status combined with MYOM2-rs2294066 precisely predicts anti-TNF response in ankylosing spondylitis. Mol Med [Internet]. 25 (2019) [cited (2020 Dec 14)]. Available from: https://molmed.biomedcentral.com/articles/10.1186/s10020-019-0093-2.

[77] Borda JP y L, Campos J, Sanz J, et al. Predictive clinical-genetic model of long-term nonresponse to tumor necrosis factor-alpha inhibitor therapy in spondyloarthritis. Int $\mathbf{J}$ Rheum Dis. 1529-153722 (2019).

[78] Ovejero-Benito MC, Reolid A, Sánchez-Jiménez P, et al. Histone modifications associated with biological drug response in moderate-to-severe psoriasis. Exp Dermatol. 1361-137127 (2018).

[79] Ciechomska M, Bonek K, Merdas M, et al. Changes in MiRNA-5196 Expression as a Potential Biomarker of Anti-TNF- $\alpha$ Therapy in Rheumatoid Arthritis and Ankylosing Spondylitis Patients. Arch Immunol Ther Exp (Warsz). 389-39766 (2018).

[80] Arends S, van der Veer E, Groen H, et al. Serum MMP-3 level as a biomarker for monitoring and predicting response to etanercept treatment in ankylosing spondylitis. J Rheumatol. 1644-165038 (2011).

[81] Wagner C, Visvanathan S, Braun J, et al. Serum markers associated with clinical improvement in patients with ankylosing spondylitis treated with golimumab. Ann Rheum Dis. 674-68071 (2012).

[82] Ademowo OS, Hernandez B, Collins E, et al. Discovery and confirmation of a protein biomarker panel with potential to predict response to biological therapy in psoriatic arthritis. Ann Rheum Dis. 234-24175 (2016). 
[83] Turina MC, Yeremenko N, Paramarta JE, et al. Calprotectin (S100A8/9) as serum biomarker for clinical response in proof-of-concept trials in axial and peripheral spondyloarthritis. Arthritis Res Ther. 41316 (2014).

[84] Østgård R, Deleuran B, Dam M, et al. Faecal calprotectin detects subclinical bowel inflammation and may predict treatment response in spondyloarthritis. Scand $\mathrm{J}$ Rheumatol. 48-5547 (2018).

[85] $\mathrm{Hu} \mathrm{H}, \mathrm{Du} \mathrm{F}$, Zhang S, et al. Serum calprotectin correlates with risk and disease severity of ankylosing spondylitis and its change during first month might predict favorable response to treatment. Mod Rheumatol. 836-84229 (2019).

[86] Jarlborg M, Courvoisier DS, Lamacchia C, et al. Serum calprotectin: a promising biomarker in rheumatoid arthritis and axial spondyloarthritis. Arthritis Res Ther. 10522 (2020).

[87] Baraliakos X, Szumski A, Koenig AS, et al. The role of C-reactive protein as a predictor of treatment response in patients with ankylosing spondylitis. Semin Arthritis Rheum. 997-100448 (2019).

[88] Hokstad I, Deyab G, Wang Fagerland M, et al. Tumor necrosis factor inhibitors are associated with reduced complement activation in spondylarthropathies: An observational study. Miyabe Y, editor. PLOS ONE. e022007914 (2019).

[89] Chimenti MS, Perricone C, Graceffa D, et al. Complement system in psoriatic arthritis: a useful marker in response prediction and monitoring of anti-TNF treatment. Clin Exp Rheumatol. 23-3030 (2012).

[90] Dolcino M, Tinazzi E, Pelosi A, et al. Gene Expression Analysis before and after Treatment with Adalimumab in Patients with Ankylosing Spondylitis Identifies Molecular Pathways Associated with Response to Therapy. Genes. 1278 (2017).

[91] Enginar AU, Kacar C. Neutrophil-lymphocyte and platelet-lymphocyte rate and their seasonal differences in ankylosing spondylitis and rheumatoid arthritis patients using anti-TNF medication. Bratisl Med J. 586-592120 (2019).

[92] Miyagawa I, Nakayamada S, Nakano K, et al. Precision medicine using different biological DMARDs based on characteristic phenotypes of peripheral T helper cells in psoriatic arthritis. Rheumatology. 336-34458 (2019).

[93] Schulte-Wrede U, Sörensen T, Grün JR, et al. An explorative study on deep profiling of peripheral leukocytes to identify predictors for responsiveness to anti-tumour necrosis factor alpha therapies in ankylosing spondylitis: natural killer cells in focus. Arthritis Res Ther [Internet]. 20 (2018) [cited (2020 Dec 14)]. Available from: https://arthritisresearch.biomedcentral.com/articles/10.1186/s13075-018-1692-y.

[94] Dulic S, Vasarhelyi Z, Bajnok A, et al. The Impact of Anti-TNF Therapy on CD4+ and CD8+ Cell Subsets in Ankylosing Spondylitis. Pathobiology. 201-21085 (2018). 
[95] Xueyi L, Lina C, Zhenbiao W, et al. Levels of Circulating Th17 Cells and Regulatory T Cells in Ankylosing Spondylitis Patients with an Inadequate Response to Anti-TNF- $\alpha$ Therapy. J Clin Immunol. 151-16133 (2013).

[96] Andersen T, Østgård RD, Aspari MP, et al. Anti-tumor necrosis factor treatment increases both the Th17 and Th22 T helper subsets in spondyloarthritis. APMIS. 789-796127 (2019).

[97] Yang M, Lv Q, Wei Q, et al. TNF- $\alpha$ inhibitor therapy can improve the immune imbalance of CD4+ T cells and negative regulatory cells but not CD8+ T cells in ankylosing spondylitis. Arthritis Res Ther. 14922 (2020).

[98] Pavy S, Toonen EJM, Miceli-Richard C, et al. Tumour necrosis factor alpha -308G->A polymorphism is not associated with response to TNFalpha blockers in Caucasian patients with rheumatoid arthritis: systematic review and meta-analysis. Ann Rheum Dis. 1022-102869 (2010).

[99] Zeng Z, Duan Z, Zhang T, et al. Association between tumor necrosis factor- $\alpha$ (TNF- $\alpha$ ) promoter $-308 \mathrm{G} / \mathrm{A}$ and response to TNF- $\alpha$ blockers in rheumatoid arthritis: a metaanalysis. Mod Rheumatol. 489-49523 (2013).

[100] Cui J, Stahl EA, Saevarsdottir S, et al. Genome-wide association study and gene expression analysis identifies CD84 as a predictor of response to etanercept therapy in rheumatoid arthritis. PLoS Genet. e10033949 (2013).

[101] Swierkot J, Bogunia-Kubik K, Nowak B, et al. Analysis of associations between polymorphisms within genes coding for tumour necrosis factor (TNF)-alpha and TNF receptors and responsiveness to TNF-alpha blockers in patients with rheumatoid arthritis. Joint Bone Spine. 94-9982 (2015).

[102] Ferreiro-Iglesias A, Montes A, Perez-Pampin E, et al. Replication of PTPRC as genetic biomarker of response to TNF inhibitors in patients with rheumatoid arthritis. Pharmacogenomics J. 137-14016 (2016).

[103] Honne K, Hallgrímsdóttir I, Wu C, et al. A longitudinal genome-wide association study of anti-tumor necrosis factor response among Japanese patients with rheumatoid arthritis. Arthritis Res Ther. 1218 (2016).

[104] Julià A, Fernandez-Nebro A, Blanco F, et al. A genome-wide association study identifies a new locus associated with the response to anti-TNF therapy in rheumatoid arthritis. Pharmacogenomics J. 147-15016 (2016).

[105] Cui J, Diogo D, Stahl EA, et al. Brief Report: The Role of Rare Protein-Coding Variants in Anti-Tumor Necrosis Factor Treatment Response in Rheumatoid Arthritis. Arthritis Rheumatol Hoboken NJ. 735-74169 (2017). 
[106] Marwa OS, Kalthoum T, Wajih K, et al. Association of IL17A and IL17F genes with rheumatoid arthritis disease and the impact of genetic polymorphisms on response to treatment. Immunol Lett. 24-36183 (2017).

[107] Sieberts SK, Zhu F, García-García J, et al. Crowdsourced assessment of common genetic contribution to predicting anti-TNF treatment response in rheumatoid arthritis. Nat Commun. 124607 (2016).

** Community-based effort to assess the use of SNP data to predict clinical outcome of TNF inhibition in RA, using machine learning algorithms. The study found that the genetic data to the prediction contributed marginally to the predictive power, compared to the clinical data.

[108] Cherlin S, Plant D, Taylor JC, et al. Prediction of treatment response in rheumatoid arthritis patients using genome-wide SNP data. Genet Epidemiol. 754-77142 (2018).

[109] Guan Y, Zhang H, Quang D, et al. Machine Learning to Predict Anti-Tumor Necrosis Factor Drug Responses of Rheumatoid Arthritis Patients by Integrating Clinical and Genetic Markers. Arthritis Rheumatol Hoboken NJ. 1987-199671 (2019).

[110] Ferreiro-Iglesias A, Montes A, Perez-Pampin E, et al. Evaluation of 12 GWAS-drawn SNPs as biomarkers of rheumatoid arthritis response to TNF inhibitors. A potential SNP association with response to etanercept. PloS One. e021307314 (2019).

[111] Bai M, He X, He Y, et al. IL-7R gene polymorphisms among patients with rheumatoid arthritis: A case-control study. Mol Genet Genomic Med. e007387 (2019).

[112] Castro-Villegas C, Pérez-Sánchez C, Escudero A, et al. Circulating miRNAs as potential biomarkers of therapy effectiveness in rheumatoid arthritis patients treated with antiTNF $\alpha$. Arthritis Res Ther. 4917 (2015).

[113] Krintel SB, Dehlendorff C, Hetland ML, et al. Prediction of treatment response to adalimumab: a double-blind placebo-controlled study of circulating microRNA in patients with early rheumatoid arthritis. Pharmacogenomics J. 141-14616 (2016).

[114] Oswald M, Curran ME, Lamberth SL, et al. Modular analysis of peripheral blood gene expression in rheumatoid arthritis captures reproducible gene expression changes in tumor necrosis factor responders. Arthritis Rheumatol Hoboken NJ. 344-35167 (2015).

[115] Huang Q-L, Zhou F-J, Wu C-B, et al. Circulating Biomarkers for Predicting Infliximab Response in Rheumatoid Arthritis: A Systematic Bioinformatics Analysis. Med Sci Monit Int Med J Exp Clin Res. 1849-185523 (2017).

[116] Byng-Maddick R, Turner CT, Pollara G, et al. Tumor Necrosis Factor (TNF) Bioactivity at the Site of an Acute Cell-Mediated Immune Response Is Preserved in Rheumatoid Arthritis Patients Responding to Anti-TNF Therapy. Front Immunol. 9328 (2017). 
[117] Shi R, Chen M, Litifu B. Serum interleukin-6 and survivin levels predict clinical response to etanercept treatment in patients with established rheumatoid arthritis. Mod Rheumatol. 126-13228 (2018).

[118] Han BK, Kuzin I, Gaughan JP, et al. Baseline CXCL10 and CXCL13 levels are predictive biomarkers for tumor necrosis factor inhibitor therapy in patients with moderate to severe rheumatoid arthritis: a pilot, prospective study. Arthritis Res Ther. 9318 (2016).

[119] Haschka J, Englbrecht M, Hueber AJ, et al. Relapse rates in patients with rheumatoid arthritis in stable remission tapering or stopping antirheumatic therapy: interim results from the prospective randomised controlled RETRO study. Ann Rheum Dis. 45-5175 (2016).

[120] Curtis JR, van der Helm-van Mil AH, Knevel R, et al. Validation of a novel multibiomarker test to assess rheumatoid arthritis disease activity. Arthritis Care Res. 1794-180364 (2012).

[121] Centola M, Cavet G, Shen Y, et al. Development of a multi-biomarker disease activity test for rheumatoid arthritis. PloS One. e606358 (2013).

[122] Hirata S, Li W, Defranoux N, et al. A multi-biomarker disease activity score tracks clinical response consistently in patients with rheumatoid arthritis treated with different anti-tumor necrosis factor therapies: A retrospective observational study. Mod Rheumatol. 344-34925 (2015).

[123] Hirata S, Li W, Kubo S, et al. Association of the multi-biomarker disease activity score with joint destruction in patients with rheumatoid arthritis receiving tumor necrosis factor-alpha inhibitor treatment in clinical practice. Mod Rheumatol. 850-85626 (2016).

[124] Li W, Sasso EH, van der Helm-van Mil AHM, et al. Relationship of multi-biomarker disease activity score and other risk factors with radiographic progression in an observational study of patients with rheumatoid arthritis. Rheumatol Oxf Engl. 35736655 (2016).

[125] Fleischmann R, Connolly SE, Maldonado MA, et al. Brief Report: Estimating Disease Activity Using Multi-Biomarker Disease Activity Scores in Rheumatoid Arthritis Patients Treated With Abatacept or Adalimumab. Arthritis Rheumatol Hoboken NJ. 2083-208968 (2016).

[126] Hambardzumyan K, Bolce RJ, Saevarsdottir S, et al. Association of a multibiomarker disease activity score at multiple time-points with radiographic progression in rheumatoid arthritis: results from the SWEFOT trial. RMD Open. e0001972 (2016).

[127] Rech J, Hueber AJ, Finzel S, et al. Prediction of disease relapses by multibiomarker disease activity and autoantibody status in patients with rheumatoid arthritis on tapering DMARD treatment. Ann Rheum Dis. 1637-164475 (2016). 
[128] Hambardzumyan K, Saevarsdottir S, Forslind K, et al. A Multi-Biomarker Disease Activity Score and the Choice of Second-Line Therapy in Early Rheumatoid Arthritis After Methotrexate Failure. Arthritis Rheumatol Hoboken NJ. 953-96369 (2017).

[129] Krabbe S, Bolce R, Brahe $\mathrm{CH}$, et al. Investigation of a multi-biomarker disease activity score in rheumatoid arthritis by comparison with magnetic resonance imaging, computed tomography, ultrasonography, and radiography parameters of inflammation and damage. Scand J Rheumatol. 353-35846 (2017).

[130] Bouman CAM, van der Maas A, van Herwaarden N, et al. A multi-biomarker score measuring disease activity in rheumatoid arthritis patients tapering adalimumab or etanercept: predictive value for clinical and radiographic outcomes. Rheumatol Oxf Engl. 973-98056 (2017).

[131] Ghiti Moghadam M, Lamers-Karnebeek FBG, Vonkeman HE, et al. Multi-biomarker disease activity score as a predictor of disease relapse in patients with rheumatoid arthritis stopping TNF inhibitor treatment. PloS One. e019242513 (2018).

[132] Bechman K, Tweehuysen L, Garrood T, et al. Flares in Rheumatoid Arthritis Patients with Low Disease Activity: Predictability and Association with Worse Clinical Outcomes. J Rheumatol. 1515-152145 (2018).

[133] Curtis JR, Brahe CH, Østergaard M, et al. Predicting risk for radiographic damage in rheumatoid arthritis: comparative analysis of the multi-biomarker disease activity score and conventional measures of disease activity in multiple studies. Curr Med Res Opin. 1483-149335 (2019).

[134] Cuppen B, Fritsch-Stork R, Eekhout I, et al. Proteomics to predict the response to tumour necrosis factor- $\alpha$ inhibitors in rheumatoid arthritis using a supervised cluster-analysis based protein score. Scand J Rheumatol. 12-2147 (2018).

[135] Choi IY, Gerlag DM, Herenius MJ, et al. MRP8/14 serum levels as a strong predictor of response to biological treatments in patients with rheumatoid arthritis. Ann Rheum Dis. 499-50574 (2015).

[136] Nair SC, Welsing PMJ, Choi IYK, et al. A Personalized Approach to Biological Therapy Using Prediction of Clinical Response Based on MRP8/14 Serum Complex Levels in Rheumatoid Arthritis Patients. PloS One. e015236211 (2016).

[137] Nordal HH, Brun JG, Hordvik M, et al. Calprotectin (S100A8/A9) and S100A12 are associated with measures of disease activity in a longitudinal study of patients with rheumatoid arthritis treated with infliximab. Scand J Rheumatol. 274-28145 (2016).

[138] Smith SL, Plant D, Eyre S, et al. The predictive value of serum S100A9 and response to etanercept is not confirmed in a large UK rheumatoid arthritis cohort. Rheumatol Oxf Engl. 1019-102456 (2017). 
[139] Inciarte-Mundo J, Ramirez J, Hernández MV, et al. Calprotectin strongly and independently predicts relapse in rheumatoid arthritis and polyarticular psoriatic arthritis patients treated with tumor necrosis factor inhibitors: a 1-year prospective cohort study. Arthritis Res Ther. 27520 (2018).

[140] Tweehuysen L, den Broeder N, van Herwaarden N, et al. Predictive value of serum calprotectin (S100A8/A9) for clinical response after starting or tapering anti-TNF treatment in patients with rheumatoid arthritis. RMD Open. e0006544 (2018).

[141] Yunchun L, Yue W, Jun FZ, et al. Clinical Significance of Myeloid-Related Protein 8/14 as a Predictor for Biological Treatment and Disease Activity in Rheumatoid Arthritis. Ann Clin Lab Sci. 63-6848 (2018).

[142] de Moel EC, Rech J, Mahler M, et al. Circulating calprotectin (S100A8/A9) is higher in rheumatoid arthritis patients that relapse within 12 months of tapering anti-rheumatic drugs. Arthritis Res Ther. 26821 (2019).

[143] Daien CI, Gailhac S, Mura T, et al. High levels of memory B cells are associated with response to a first tumor necrosis factor inhibitor in patients with rheumatoid arthritis in a longitudinal prospective study. Arthritis Res Ther. R9516 (2014).

[144] Citro A, Scrivo R, Martini H, et al. CD8+ T Cells Specific to Apoptosis-Associated Antigens Predict the Response to Tumor Necrosis Factor Inhibitor Therapy in Rheumatoid Arthritis. PloS One. e012860710 (2015).

[145] Hull DN, Cooksley H, Chokshi S, et al. Increase in circulating Th17 cells during antiTNF therapy is associated with ultrasonographic improvement of synovitis in rheumatoid arthritis. Arthritis Res Ther. 30318 (2016).

[146] Salomon S, Guignant C, Morel P, et al. Th17 and CD24hiCD27+ regulatory B lymphocytes are biomarkers of response to biologics in rheumatoid arthritis. Arthritis Res Ther. 3319 (2017).

[147] Lee H-N, Kim Y-K, Kim G-T, et al. Neutrophil-to-lymphocyte and platelet-tolymphocyte ratio as predictors of 12 -week treatment response and drug persistence of anti-tumor necrosis factor- $\alpha$ agents in patients with rheumatoid arthritis: a retrospective chart review analysis. Rheumatol Int. 859-86839 (2019).

[148] Cianciotti BC, Ruggiero E, Campochiaro C, et al. CD4+ Memory Stem T Cells Recognizing Citrullinated Epitopes Are Expanded in Patients With Rheumatoid Arthritis and Sensitive to Tumor Necrosis Factor Blockade. Arthritis Rheumatol Hoboken NJ. $565-57572$ (2020).

[149] Rodríguez-Martín E, Nieto-Gañán I, Hernández-Breijo B, et al. Blood Lymphocyte Subsets for Early Identification of Non-Remission to TNF Inhibitors in Rheumatoid Arthritis. Front Immunol. 191311 (2020). 
[150] Bystrom J, Clanchy FI, Taher TE, et al. Response to Treatment with TNF $\alpha$ Inhibitors in Rheumatoid Arthritis Is Associated with High Levels of GM-CSF and GM-CSF+ T Lymphocytes. Clin Rev Allergy Immunol. 265-27653 (2017).

[151] Lliso-Ribera G, Humby F, Lewis M, et al. Synovial tissue signatures enhance clinical classification and prognostic/treatment response algorithms in early inflammatory arthritis and predict requirement for subsequent biological therapy: results from the pathobiology of early arthritis cohort (PEAC). Ann Rheum Dis. 1642-165278 (2019).

[152] Massey J, Plant D, Hyrich K, et al. Genome-wide association study of response to tumour necrosis factor inhibitor therapy in rheumatoid arthritis. Pharmacogenomics J. 657-66418 (2018).

[153] Farutin V, Prod'homme T, McConnell K, et al. Molecular profiling of rheumatoid arthritis patients reveals an association between innate and adaptive cell populations and response to anti-tumor necrosis factor. Arthritis Res Ther. 21621 (2019).

* Combines whole blood transcriptome and plasma proteome analysis of RA patients to identify molecular signatures of anti-TNF treatment.

[154] Tao W, Concepcion AN, Vianen M, et al. Multiomics and Machine Learning Accurately Predict Clinical Response to Adalimumab and Etanercept Therapy in Patients With Rheumatoid Arthritis. Arthritis Rheumatol Hoboken NJ. (2020).

* This paper explores the ability of random forest-based algorithms to model gene expression and DNA methylation data in order to predict therapeutic responses in RA.

[155] Spiliopoulou A, Colombo M, Plant D, et al. Association of response to TNF inhibitors in rheumatoid arthritis with quantitative trait loci for CD40 and CD39. Ann Rheum Dis. 1055-106178 (2019).

** Analysis of genotypic scores for RA associated loci, for immune cell traits, and for gene expression or methylation to construct predictive models for response to TNF inhibitors.

[156] Folkersen L, Brynedal B, Diaz-Gallo LM, et al. Integration of known DNA, RNA and protein biomarkers provides prediction of anti-TNF response in rheumatoid arthritis: results from the COMBINE study. Mol Med Camb Mass. 322-32822 (2016).

** This study exemplifies the increased power resulting from the integration of different types of biomarkers (transcript levels, proteins, SNPs) in predictive models of therapeutic response in RA.

[157] Tasaki S, Suzuki K, Kassai Y, et al. Multi-omics monitoring of drug response in rheumatoid arthritis in pursuit of molecular remission. Nat Commun. 27559 (2018).

[158] Wang M-H, Friton JJ, Raffals LE, et al. Novel Genetic Risk Variants Can Predict AntiTNF Agent Response in Patients With Inflammatory Bowel Disease. J Crohns Colitis. 1036-104313 (2019). 
[159] Gomez A, Ingelman-Sundberg M. Pharmacoepigenetics: Its Role in Interindividual Differences in Drug Response. Clin Pharmacol Ther. 426-43085 (2009).

[160] Cherqaoui B, Crémazy F, Hue C, et al. Epigenetics of spondyloarthritis. Joint Bone Spine. 565-57187 (2020).

[161] Aslani S, Mahmoudi M, Karami J, et al. Epigenetic alterations underlying autoimmune diseases. Autoimmunity. 69-8349 (2016).

[162] Toonen EJM, Gilissen C, Franke B, et al. Validation study of existing gene expression signatures for anti-TNF treatment in patients with rheumatoid arthritis. PloS One. e331997 (2012).

[163] Reinhardt A, Prinz I. Whodunit? The Contribution of Interleukin (IL)-17/IL-22Producing $\gamma \delta$ T Cells, $\alpha \beta$ T Cells, and Innate Lymphoid Cells to the Pathogenesis of Spondyloarthritis. Front Immunol. 8859 (2018).

[164] Tsukazaki H, Kaito T. The Role of the IL-23/IL-17 Pathway in the Pathogenesis of Spondyloarthritis. Int J Mol Sci. 640121 (2020).

[165] Huang Y, Deng W, Zheng S, et al. Relationship between monocytes to lymphocytes ratio and axial spondyloarthritis. Int Immunopharmacol. 43-4657 (2018).

[166] Uslu AU, Küçük A, Şahin A, et al. Two new inflammatory markers associated with Disease Activity Score-28 in patients with rheumatoid arthritis: neutrophil-lymphocyte ratio and platelet-lymphocyte ratio. Int J Rheum Dis. 731-73518 (2015).

[167] Mercan R, Bitik B, Tufan A, et al. The Association Between Neutrophil/Lymphocyte Ratio and Disease Activity in Rheumatoid Arthritis and Ankylosing Spondylitis. J Clin Lab Anal. 597-60130 (2016).

[168] Nishida Y, Hosomi S, Yamagami H, et al. Neutrophil-to-Lymphocyte Ratio for Predicting Loss of Response to Infliximab in Ulcerative Colitis. PLOS ONE. e016984512 (2017).

[169] Martin JC, Chang C, Boschetti G, et al. Single-Cell Analysis of Crohn's Disease Lesions Identifies a Pathogenic Cellular Module Associated with Resistance to Anti-TNF Therapy. Cell. 1493-1508.e20178 (2019).

[170] Lorenzin M, Ometto F, Ortolan A, et al. An update on serum biomarkers to assess axial spondyloarthritis and to guide treatment decision. Ther Adv Musculoskelet Dis. 1759720X209342712 (2020).

[171] Bal A, Unlu E, Bahar G, et al. Comparison of serum IL-1 beta, sIL-2R, IL-6, and TNFalpha levels with disease activity parameters in ankylosing spondylitis. Clin Rheumatol. 211-21526 (2007). 
[172] Arends S, van der Veer E, Kallenberg CGM, et al. Baseline predictors of response to TNF- $\alpha$ blocking therapy in ankylosing spondylitis. Curr Opin Rheumatol. 290-29824 (2012).

[173] Maksymowych WP. Biomarkers for Diagnosis of Axial Spondyloarthritis, Disease Activity, Prognosis, and Prediction of Response to Therapy. Front Immunol [Internet]. 10 (2019) [cited (2020 Nov 1)]. Available from: https://www.frontiersin.org/articles/10.3389/fimmu.2019.00305/full.

[174] Fleischmann R. Value of the Multibiomarker Disease Activity Score to Predict Remission in RA: What Does the Evidence Show? J Rheumatol. 443-44646 (2019).

[175] Calabrese LH. MBDA: A Valuable Tool for Medical Decision Making. J Rheumatol. 164246 (2019).

[176] Masi AT. What further data are needed to value the Multi-Biomarker Disease Activity score for measuring rheumatoid arthritis disease activity: comment on the article by Johnson et al. Arthritis Care Res. 1339-134072 (2020).

[177] van der Helm-van Mil AHM, Knevel R, Cavet G, et al. An evaluation of molecular and clinical remission in rheumatoid arthritis by assessing radiographic progression. Rheumatol Oxf Engl. 839-84652 (2013).

[178] Romand X, Bernardy C, Nguyen MVC, et al. Systemic calprotectin and chronic inflammatory rheumatic diseases. Joint Bone Spine. 691-69886 (2019).

[179] Austermann J, Spiekermann C, Roth J. S100 proteins in rheumatic diseases. Nat Rev Rheumatol. 528-54114 (2018).

[180] Sun S, Bay-Jensen A-C, Karsdal MA, et al. The active form of MMP-3 is a marker of synovial inflammation and cartilage turnover in inflammatory joint diseases. BMC Musculoskelet Disord. 9315 (2014).

[181] Gao JW, Zhang KF, Lu JS, et al. Serum matrix metalloproteinases-3 levels in patients with ankylosing spondylitis. Genet Mol Res GMR. 17068-1707814 (2015).

[182] Mou Y-K, Zhang P-P, Li Q-X, et al. Changes of serum levels of MMP-3, sRANKL, and OPG in juvenile-onset ankylosing spondylitis patients carrying different HLA-B27 subtypes. Clin Rheumatol. 1085-108934 (2015).

[183] Jadon DR, Sengupta R, Nightingale A, et al. Serum bone-turnover biomarkers are associated with the occurrence of peripheral and axial arthritis in psoriatic disease: a prospective cross-sectional comparative study. Arthritis Res Ther. 21019 (2017).

[184] Moz S, Aita A, Basso D, et al. Spondyloarthritis: Matrix Metalloproteinasesas Biomarkers of Pathogenesis and Response to Tumor Necrosis Factor (TNF) Inhibitors. Int J Mol Sci. 83018 (2017). 
[185] Pedersen SJ, Hetland ML, Sørensen IJ, et al. Circulating levels of interleukin-6, vascular endothelial growth factor, YKL-40, matrix metalloproteinase-3, and total aggrecan in spondyloarthritis patients during 3 years of treatment with TNFa inhibitors. Clin Rheumatol. 1301-130929 (2010).

[186] Chandran V, Shen H, Pollock RA, et al. Soluble Biomarkers Associated with Response to Treatment with Tumor Necrosis Factor Inhibitors in Psoriatic Arthritis. J Rheumatol. 866-87140 (2013).

[187] Bertani L, Fornai M, Fornili M, et al. Serum oncostatin M at baseline predicts mucosal healing in Crohn's disease patients treated with infliximab. Aliment Pharmacol Ther. 284-29152 (2020).

[188] Minar P, Lehn C, Tsai Y-T, et al. Elevated Pretreatment Plasma Oncostatin M Is Associated With Poor Biochemical Response to Infliximab. Crohns Colitis 360 [Internet]. 1 (2019) [cited (2020 Dec 10)]. Available from: https://academic.oup.com/crohnscolitis360/article/1/3/otz026/5551502.

[189] West NR, Hegazy AN, Owens BMJ, et al. Oncostatin M drives intestinal inflammation and predicts response to tumor necrosis factor-neutralizing therapy in patients with inflammatory bowel disease. Nat Med. 579-58923 (2017).

[190] West NR. Coordination of Immune-Stroma Crosstalk by IL-6 Family Cytokines. Front Immunol [Internet]. 10 (2019) [cited (2020 Dec 15)]. Available from: https://www.frontiersin.org/articles/10.3389/fimmu.2019.01093/full.

** OSM and OSMR are overexpressed in the majority of active IBD mucosa, particularly in patients resistant to anti-TNF. OSM could therefore be a novel predictive biomarker for anti-TNF and therapeutic target for this clinically challenging population. 


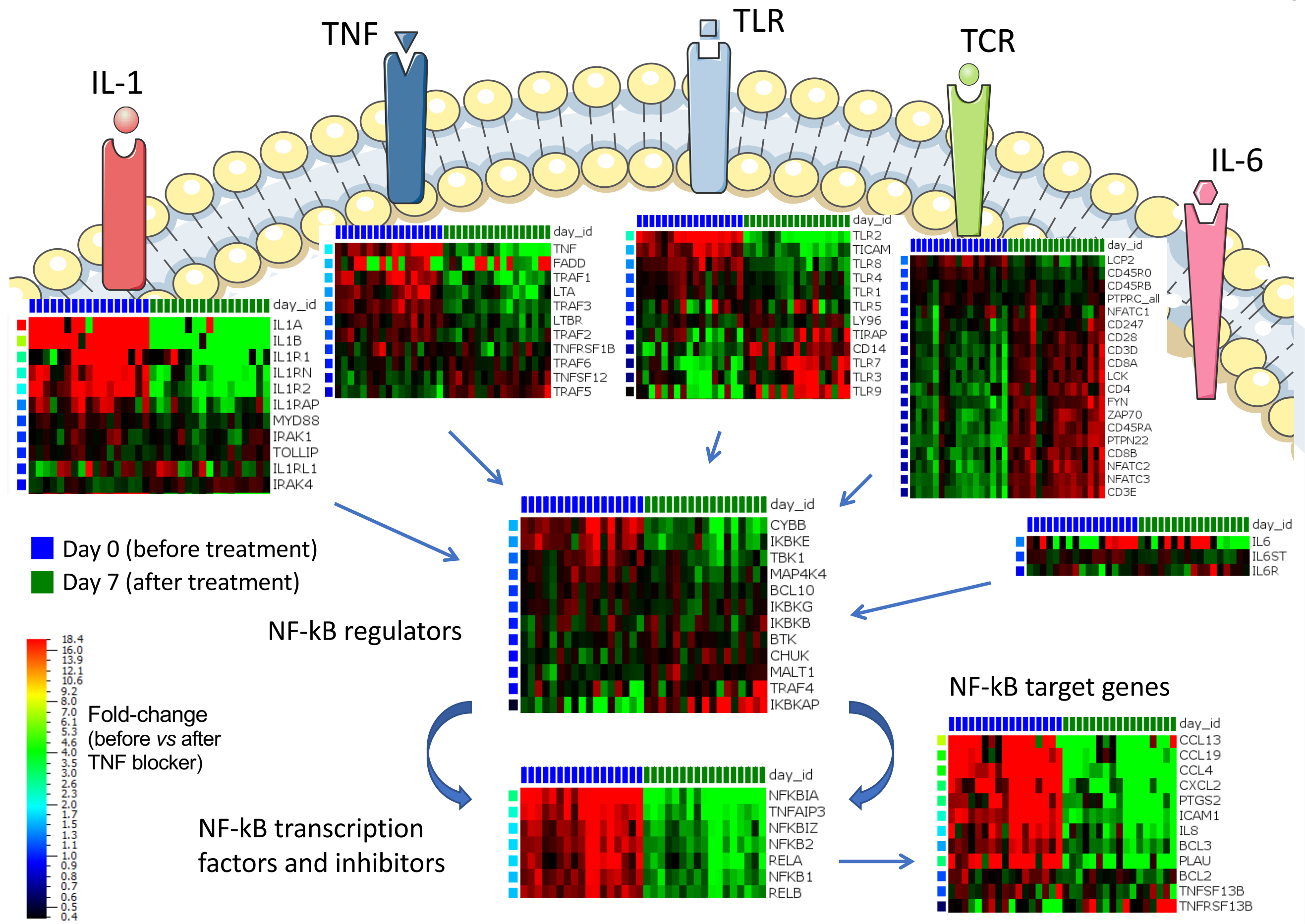


Candida stimulation

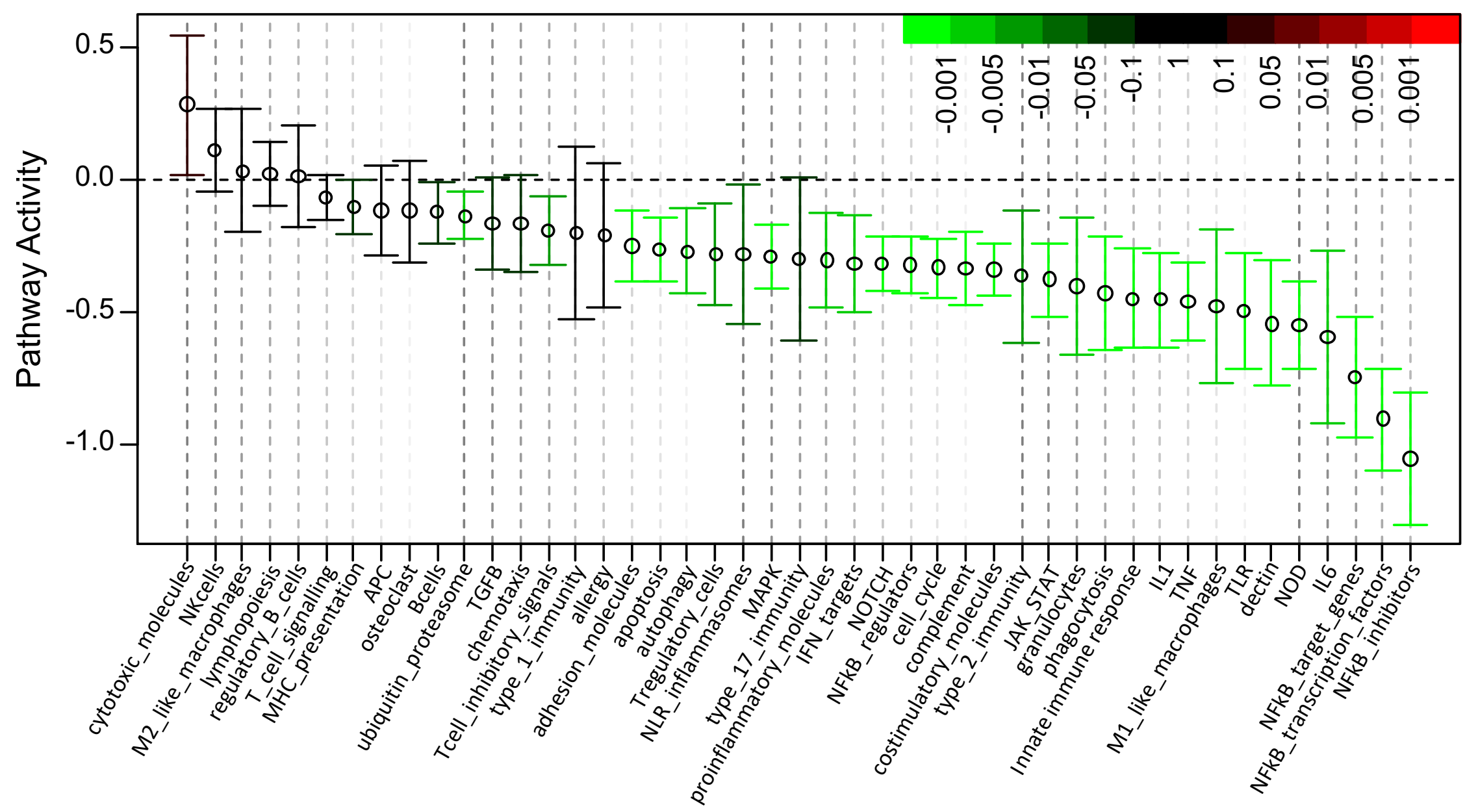


A

Cytotoxic molecules

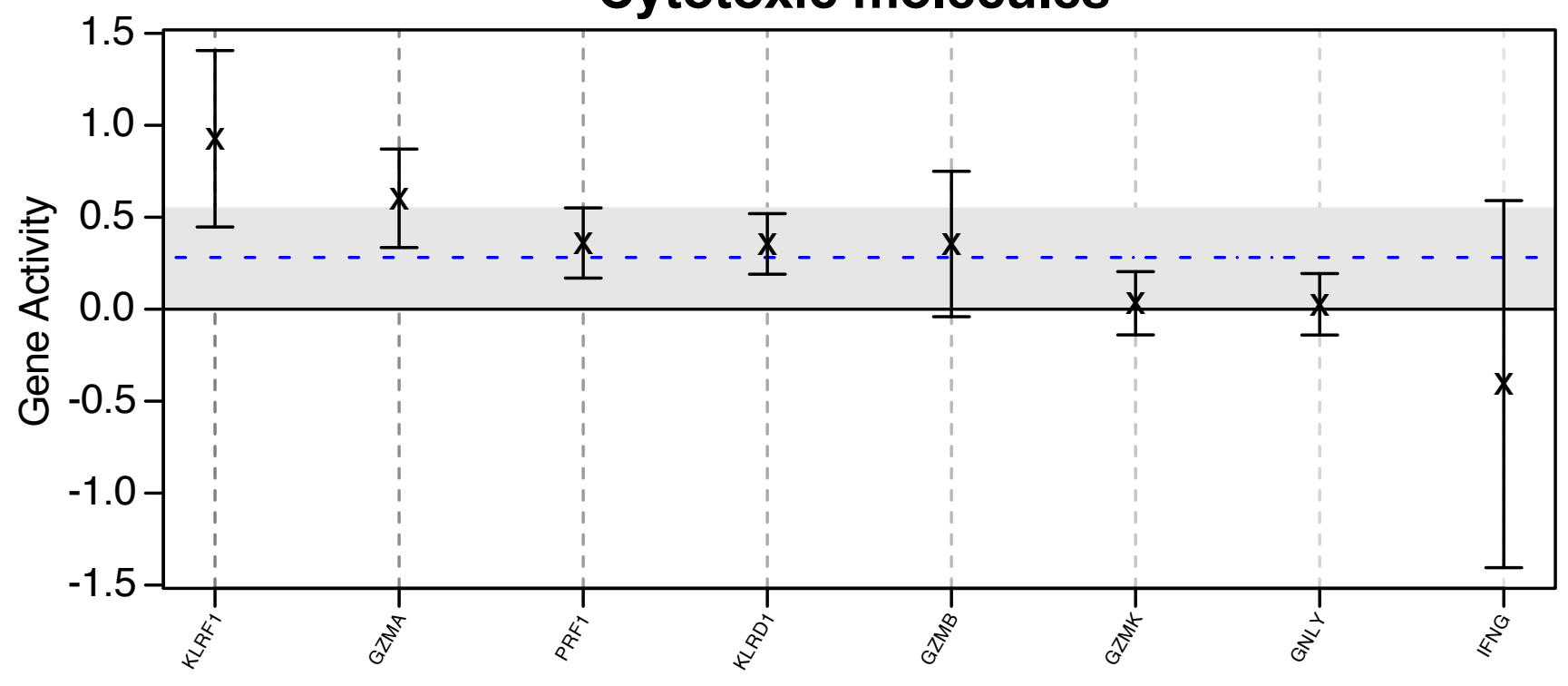

B

M2 macrophages

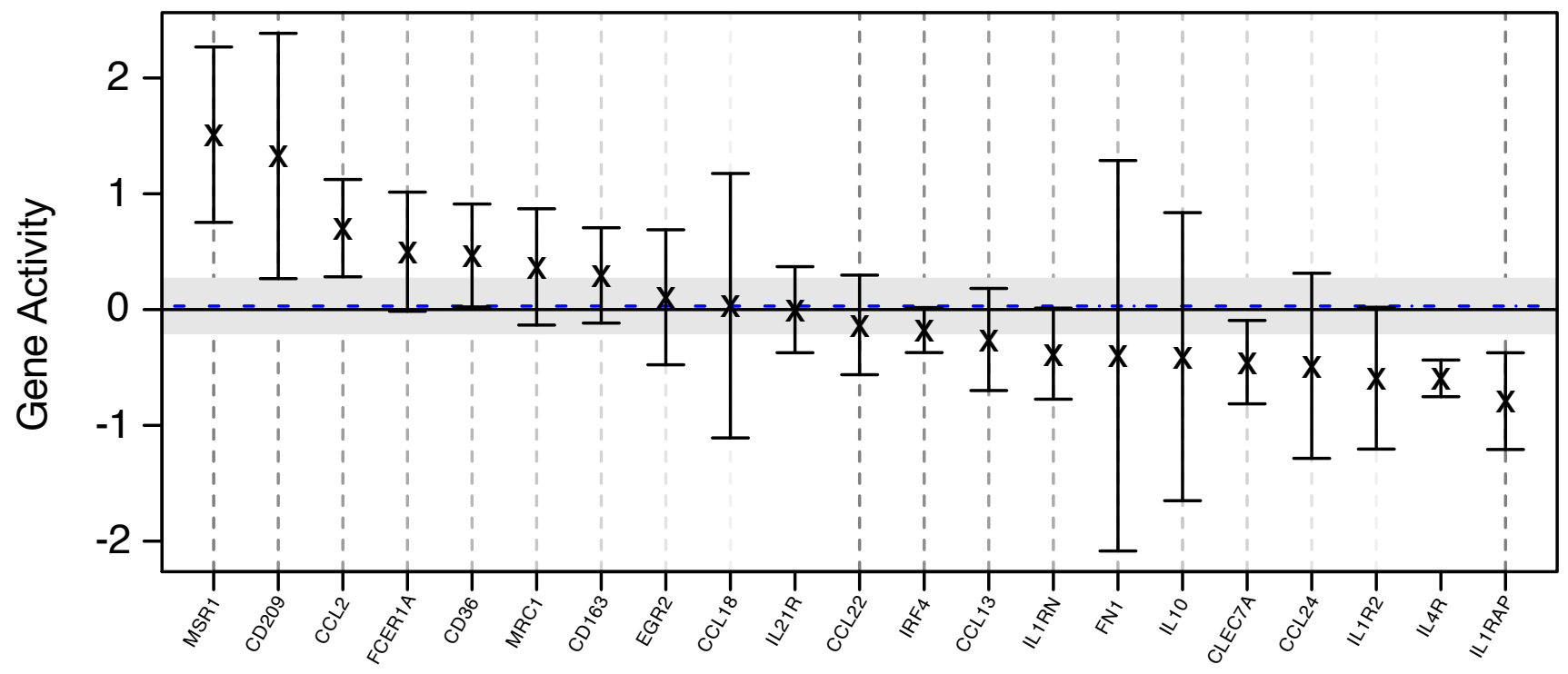

C Proinflammatory molecules

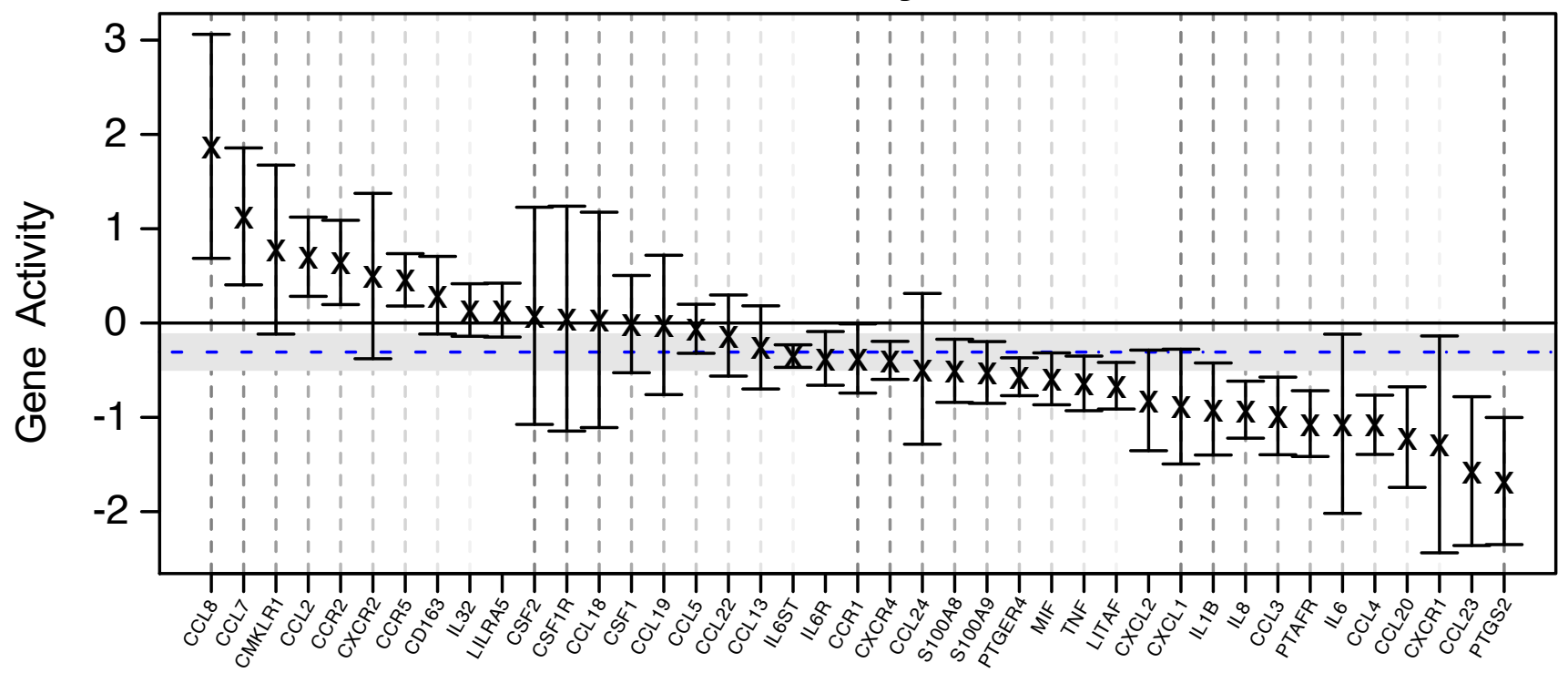


Figure 4

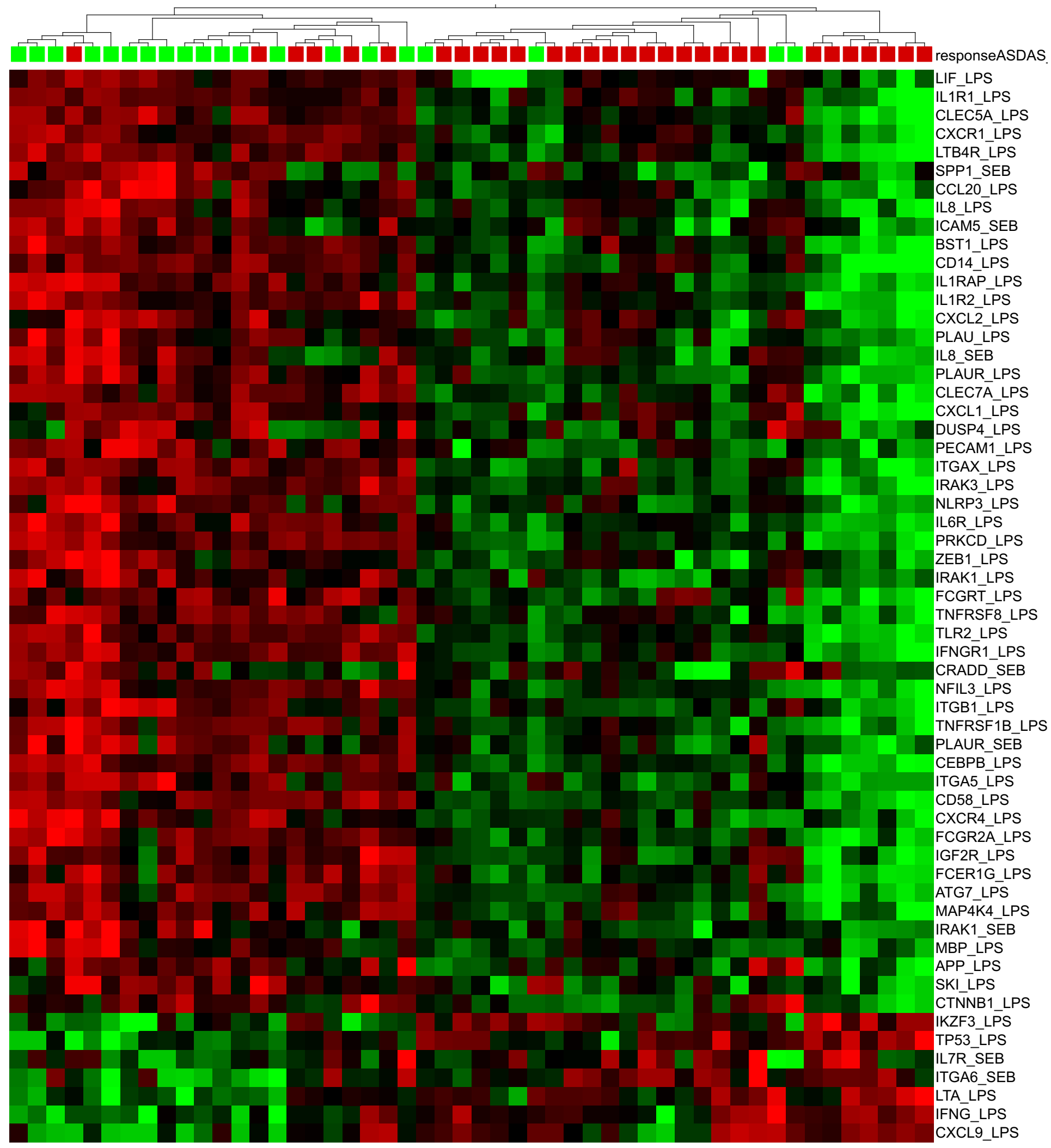

